\title{
Fiat Money, Intrinsic Properties, and Government Transaction Policy
}

\author{
Xavier Cuadras-Morató \\ Department of Economics \\ Universitat Pompeu Fabra
}

Phone: 34935422606

Fax: 34935421746

E-mail: xavier.cuadras@econ.upf.es

${ }^{*}$ Thanks to Randall Wright for very useful comments and suggestions. All errors are mine. 
Running Head: Money Properties and Government Policy

Corresponding Author: Xavier Cuadras-Morató

Department of Economics - Universitat Pompeu Fabra

Ramon Trias Fargas, 25-27

08005 Barcelona

Spain

Phone: 34935422606

Fax: 34935421746

E-mail: xavier.cuadras@econ.upf.es 


\begin{abstract}
Why do people coordinate on the use of valueless pieces of paper as generally accepted money? A possible answer is that these objects have intrinsic properties that make them better candidates to be used as media of exchange. Another answer stresses the fact that unconvertible fiat money will not easily appear unless there is a centralized institution that favors its use. The main objective of the paper is to analyze these questions. In order to do this, we take a model of commodity money in which fiat money does not play any significant role and modify it to examine under which circumstances fiat money might come to circulate as medium of exchange. Some of the results obtained from the model differ in a rather substantial way from previous related literature.
\end{abstract}

Keywords: Fiat Money, Government Policy, Search Theory. 


\section{Introduction}

Money circulates in the economy because many people coordinate on the use of one or several objects for exchange purposes. Thus, the choice of which object will be used as money is the final result of the behavior of many individual agents making optimal decisions on their exchange operations. Then, a natural question that arises is why people coordinate on the use of valueless pieces of paper as money. A possible answer to this question is that these objects have intrinsic properties (namely storability, homogeneity, durability, etc.) which make them better candidates to be used as money. ${ }^{1}$ Another answer, not necessarily contradictory with the first, but of very different nature, stresses the fact that non-convertible fiat money will not easily appear unless there is a centralized institution (e.g. the government) which favors its use. Declaring fiat money legal tender and accepting it for payment of taxes are examples of possible ways in which governments can favor the use of this type of money. ${ }^{2}$ The main objective of the paper is to analyze these and related questions. In order to do this, we propose the following

\footnotetext{
${ }^{1}$ Classical accounts of the desirable properties of money can be found in Jevons (1875) and Menger (1892).

${ }^{2}$ Other forms of pressure can be less subtle although maybe more effective. Thus, Chown (1994) describes how the government in revolutionary France had "remarkable success in putting so much new paper into circulation without a sharp fall in value" (Chown 1994, p. 226). The reason for this was, according to him, "the remarkable power of the central government led by the Committee of Public Safety in enforcing its dictates through the Reign of Terror" (Chown 1994, p. 226). A contemporary writer, D'Ivernois, wrote "the Guillotine, which Robespierre is said to have called an engine for coining
} 
exercise: take a model of commodity money in which fiat money does not play any significant role and, then, modify it to examine under which circumstances fiat money might come to be valued. We hope, with this exercise, to gain a better understanding of the nature of fiat money and the circumstances that explain its emergence in certain historical scenarios.

The model from which we choose to start our analysis is the commodity money model of Cuadras-Morató and Wright (1997). This is a particular version of the search theoretical model of money ${ }^{3}$ in which goods differ by the number of their potential producers ("supply") and the probability with which agents want to consume them ("demand"). In equilibrium, goods are ranked according to their relative acceptability in trading and this determines endogenously their respective “ results of the model is that fiat money never will take on value. This is because what mainly drives acceptability of goods is their final consumption value and, consequently, a good which is not valued for consumption (e.g., intrinsically useless fiat money) is the least valued of all and, so, it is the least accepted. In the current paper, our modeling strategy will be to depart from this environment and introduce modifications that will give rise to valuable fiat money. Thus, we present a version of the model in CuadrasMorató and Wright (1997) in which agents are specialized in producing one good, but are generalists in consumption. In particular, all agents have tastes that change over time according to some common distribution and this determines their preferences for the different commodities. There are two obvious ways to modify the original set up to give

\footnotetext{
${ }^{3}$ Models of the exchange process based on search theory have been used extensively to analyze the features of objects that make them more or less likely to emerge as money in equilibrium (the seminal reference here is Kiyotaki and Wright 1989).
} 
rise to valuable fiat money. First, we introduce fiat money with storage costs (or any other "intrinsic" characteristic) different from consumption goods. We show that fiat money can only take on value if and only if it has strictly better intrinsic properties than the rest of commodities. This is a result which contrasts with previous related literature such as Kiyotaki and Wright (1991) and Aiyagari and Wallace (1992), which prove that there exist equilibria in which fiat money is accepted even if it is not an object with better intrinsic properties than the rest of goods. Second, we present the same model adding government transaction policy, which may favor the use of fiat money as medium of exchange. The main result now is that fiat money might take on value depending on the values of parameters, but will never dominate as medium of exchange the best valued consumption good (e.g. gold). This result is different from those obtained in the literature about fiat money and government transaction policy. For instance, Aiyagari and Wallace (1997) and Li and Wright (1998) show that there are equilibria in which fiat money dominates all commodities, while this does not happen in our model, where fiat money cannot be better than gold (if gold was the best valued good). Consequently, one of the main contributions of this paper is to generate results that are significantly different from what has been proposed in previous literature. Interestingly, this is done in the context of a very similar search model which has been extended in a natural way, modifying the special assumption that agents specialize in both consumption and production in order to have agents who are generalists in consumption.

Inconvertible fiat money being the only generally accepted medium of exchange is something characteristic of our current monetary system. Nevertheless, early experiences of fiat money demonstrate that in different historical situations inconvertible fiat money could circulate along with commodity money (e.g. gold) without dominating it. This is 
something our model predicts: inconvertible fiat money arises if there is some centralized institution which "promotes" it through some form of transaction policy; and, moreover, it is never preferred to gold, which is accepted by everyone. ${ }^{4}$ In the absence of some sort of government policy that supported it, inconvertible fiat money would only emerge if and only if it had better intrinsic properties than commodity money. Nevertheless, it is fairly intuitive that this would hardly be the case for pieces of paper issued by private bankers or firms, with little built credibility and subject to all possible types of uncertainty and manipulation. In fact, we only observe circulation of paper money issued by private banks when there exist a convertibility clause that guarantees its value (the "free banking" experiences $)^{5}$. One of the contributions of this paper is to construct a model in which inconvertible fiat money circulates together with commodity money without dominating it. This is something we see in the first historical episodes in which non-convertible paper

\footnotetext{
${ }^{4}$ Apart from earlier episodes in China, the first example we have notice of circulating inconvertible paper money was in Sweden from 1661 to 1667 (see The Economist 1999). Chown (1994) describes in detail several other episodes of early inconvertible fiat money: John Law's experiments in France (1716-1720), the American Revolution, the French Revolution and the "assignats", the suspension of payments in England during the Napoleonic Wars (1797-1821), and the American Civil War and the greenbacks. All of them share these two characteristics: they were promoted by the government (there is not inconvertible fiat money issued by private banks, of course); and they are examples of coexistence of acceptable fiat money and commodity money (gold) in which gold always dominates fiat money as the preferred asset.
}

\footnotetext{
5 Friedman and Schwartz (1986, p.45) report that they "do not know... of any example of the private production of purely inconvertible fiat moneys".
} 
money was issued by the government and its circulation promoted by some sort of transaction policy (see note 4).

The rest of the paper is organized as follows. Section 2 describes a general model with fiat money and storage costs. Section 3 considers a simple example of that economy with two consumption goods and derives some general results. Section 4 introduces the role of government transaction policy and Sections 5 and 6 derive some results for tractable versions of this new economy. Section 7 concludes with a summary of the arguments and offers suggestions for future research.

\section{A General Model with Fiat Money and Storage Costs}

This section describes a general version of the three-commodity model of Cuadras-Morató and Wright (1997), including fiat money and storage costs. The economy is populated by a $[0,1]$ continuum of infinite-lived agents. There are $n+1$ different objects: $n$ consumption goods, each of which we shall denote by good $i(i=1,2, \ldots, n)$, and fiat money, a valueless piece of paper which cannot be consumed and has no use in production processes. All objects are indivisible ${ }^{6}$ and identically durable, homogeneous, portable, etc. The only difference is that while consumption goods can be stored by all

\footnotetext{
${ }^{6}$ This assumption conveniently simplifies the analysis of this type of models. On the other hand, it implies that the rates of exchange of all commodities are fixed to one, and makes these models ill suited to analyze prices. Trejos and Wright (1995), Shi (1995) and Li and Wright (1998) relax this assumption and deal with prices in an explicit way.
} 
agents only at some cost in terms of utility, $c_{1}$, fiat money is storable by everyone at cost $c_{0}{ }^{78}$ There are $n$ different types of agents according to the commodity that they are specialized in producing. Agents of type $i$ produce good $i$ at some common cost, $D$. We shall denote by $\sigma_{i}\left(\sum \sigma_{i}=1\right)$ the fraction of agents who are of type $i$. Agents are generalists in consumption. Thus, at every date, $t=0,1,2, \ldots$, each agent gets a taste shock (independent across agents and time) that determines the good she desires that period. We shall denote by $\delta_{i}\left(\sum_{i} \delta_{i}=1\right)$ the probability that an agent desires good $i$ at any particular period of time. If a trader desires good $i$, she gets utility $U$ from consuming good $i$ and zero utility from consuming any other good different from $i$. After consuming a good, traders immediately produce one unit of their own production commodity. We shall denote by $u=U-D$, the net utility of the joint action of consuming plus producing.

There is a fixed stock of indivisible units of fiat money in the economy, $m$ ( $m$ is also the proportion of all agents in the economy who hold one unit of fiat money). At the initial date every agent is endowed with a single unit of an object. In particular, we shall assume that a proportion 1- $m$ of agents is endowed with a unit of the good they produce, while the rest, $m$, are endowed with fiat money. All agents have exactly the same probability of getting fiat money, independently of their production type. Following Aiyagari and Wallace (1992) and Kiyotaki and Wright (1993), we assume that agents cannot produce unless they have consumed. Thus, every agent will be always holding one

\footnotetext{
${ }^{7}$ Details about the way this storage costs are incorporated into the model can be found below when the payoff functions for the agents are made explicit.

${ }^{8}$ Storage costs of fiat money could be loosely interpreted in a variety of ways such as inflation, uncertainty about future value of notes, etc. (see Li and Wright 1998 for a similar interpretation of this parameter).
} 
unit of an object and the initial stock of money is kept invariant. The sequence of events will be as follows: every period of time, agents start with some object in storage (a consumption good or fiat money). Then, they get a taste shock. If they happen to store the good they want to consume, then they consume it, produce a new good and wait for next period. Otherwise, they enter a trading process (market) in which they will be randomly matched with other traders in their same situation. Once matched, the agents will have to decide whether they want to trade or not. If they want to trade, then they swap inventories one-for-one. Whenever an agent gets the good she desires, she consumes it and immediately produces a new good; otherwise, she keeps the object she obtained and waits for next period. If they do not want to trade, then they part company and wait for the next period.

The strategic decision of traders is very simple in the economy described so far. At a given period of time, agents in the market must be holding one of two things: fiat money or some good they do not want to consume today (they would obviously never go to the market holding the good they want to eat). Then, they will be paired with another agent who will offer them one of the two following possibilities: either the good they want to consume today (which will be accepted and consumed immediately) or another object which they do not want to eat today either. Clearly, the trade decision to be taken by agents in our environment is simply this choice between two objects that are not desired for immediate consumption (one of which might be fiat money).

In order to analyze the strategic decision of agents in this economy, let $V_{i j}$ be the value function for a type- $i$ individual $(i=1, \ldots, n)$, at the end of a period holding good $j$ other than the one currently desired for consumption $(j=0,1, \ldots n$; good 0 here is simply the 
notation used for fiat money). $V_{i j}$ can be interpreted as the value of good $j$ as asset. The structure of taste shocks that we have assumed guarantees that $V_{i j}$ does not depend on the good that is desired in the current period. Logically, the strategic problem of agents can be formalized in the following way: an agent of type $i$ wants to accept good $h$ in exchange for good $j$ iff $V_{i h}>V_{i j}$ (that is, if the value of good $h$ as asset is larger than the value of $\operatorname{good} j$ ). Iff $V_{i j}>V_{i h}$, then agents do not want to accept good $h$ in exchange for good $j$ and, finally, iff $V_{i h}=V_{i j}$, then agents of type $i$ are indifferent between holding $\operatorname{good} j$ or $\operatorname{good} h$. In the particular economy we study, we can characterize the behavior of agents of type $i$ simply by ranking the $n+1$ value functions. This can be represented by a strategy vector of $[(\mathrm{n}+1) \mathrm{n}] / 2$ elements $s(i)=\left(\ldots, s(i)_{h j}, \ldots\right)$ where $s(i)_{h j}$ is defined as follows:

$$
s(i)_{h j} \in\left\{\begin{array}{c}
\{1\} \text { iff } V_{i j}>V_{i h} \\
(0,1) \text { iff } V_{i j}=V_{i h} \\
\{0\} \text { iff } V_{i j}<V_{i h}
\end{array}\right.
$$

The structure of this economy is such that it is optimal for all types of traders to use the same trading strategies. This is equivalent to saying that all agents will rank all objects in exactly the same way, independently of their production type. The reason for this is simply that, independently on the good they produce, all agents draw their taste shocks from a common distribution and, in order to evaluate the value of holding a particular object, what matters is simply the chance of consuming in future periods. In this particular sense, this is a representative agent model, where all agents are identical from the point of view of which trading strategies are optimal. In other words, the heterogeneity of agents in this model derives from the fact that they produce different sorts of commodities, but this feature does not affect the nature of the strategic problem they face. In order to avoid unnecessary repetitions, we do not provide the formal proof of this 
result here and refer the reader to Proposition 1 in Cuadras-Morató and Wright (1997), where this result has been proven in the context of a model with three commodity goods and no fiat money, but otherwise identical. This is an important feature of the model: it allows us to summarize the strategic behavior of all agents by a single vector, $\boldsymbol{s}$. In fact, without this structure of representative agent there would be little hope of solving the model.

In order to continue the analysis, we need to specify some notation. Let $p_{i j}$ denote the measure of type $i$ agents with good $j$ at the start of a period $(i=1,2, \ldots n$ and $j=0,1,2, \ldots, n),\left(\sum_{j} p_{i j}=\sigma_{i}\right)$. Let $\boldsymbol{p}=\left(\ldots p_{i j}, \ldots\right)$. The measure of agents who hold good $j$ is $P_{j}=\sum_{i j} p_{i j}{ }^{9}$ The total number of agents with good $i$ who go to the market is $\left(1-\delta_{i}\right) P_{i}$ and the total number of agents who go to the market is $N=\sum_{i}\left[\left(1-\delta_{i}\right) P_{i}\right]+P_{0}$. Let $\pi_{j i}=P_{j} \delta_{i} / N$ be the probability of meeting someone in the market who is holding good $j$ and wants to consume good $i$. Given $\boldsymbol{s}$, the distribution $\boldsymbol{p}$ evolves according to some law of motion $\boldsymbol{p}$ ' $=f(\boldsymbol{p} ; \boldsymbol{s})$. A steady-state is a solution to $\boldsymbol{p}=f(\boldsymbol{p} ; \boldsymbol{s})$. Once we know $\boldsymbol{p}$, we can determine the steady-state value of $\boldsymbol{\pi}=\left(\ldots, \pi_{j i}, \ldots\right)$ as a function of $\boldsymbol{s}$ [given the economy described by the parameters $m, \boldsymbol{\delta}=\left(\ldots, \delta_{i}, \ldots\right)$ and $\left.\boldsymbol{\sigma}=\left(\ldots, \sigma_{i}, \ldots\right)\right]$

Money in this economy is any object that is accepted by an agent not to be consumed immediately, but because of its relative high value as asset. There are two kinds of money: fiat and commodity money. As we shall see, in equilibrium all objects

\footnotetext{
${ }^{9}$ Note that $P_{0}=m$.
} 
will be ranked from more acceptable to less acceptable, reflecting their relative liquidity (or moneyness) in the economy.

In the following lines we present some results for a simple example with $n=2$ and discuss the way these are general results.

\section{An Example: Two Consumption Goods and Fiat Money}

The model described in the previous section is simplified by taking $n=2$. There are now two consumption goods and types of agents. In contrast to the general model described above, commodity money is now ruled out (e.g., no agent of type 1 (2) will ever accept good 2 (1) unless she wants to consume it immediately). Suppose an agent of type 1 who eventually produces good 1 . Then, she might want to consume it with probability $\boldsymbol{\delta}_{1}=\boldsymbol{\delta}$, producing good 1 again afterwards, or she might want to consume good 2 with probability $\delta_{2}=1-\delta$ and then go to the market. Once in the market, she might be offered good 2 by someone who wants to consume good 1, which means that trade will take place. She could also be offered good 1, which she will refuse. Finally, she could be offered fiat money. If she accepts fiat money, she will not trade it unless it is for the good that she wants to eat. ${ }^{10}$ Clearly, type 1 agents will never accept good 2 to keep it in storage. Hence, the strategic decision in this economy is reduced to a choice between

\footnotetext{
${ }^{10}$ This is so if we restrict the analysis to pure-strategies. Suppose an agent holding fiat money wishes to eat good 1 and meets with another agent holding good 2 (and then desiring good 1). They would trade with probability $\mathrm{s}_{02}\left(1-\mathrm{s}_{02}\right)=0$.
} 
one's production good and fiat money. Thus, the behavior of all agents can be summarized by a vector $\boldsymbol{s}=\left(s_{01}, s_{02}\right)$. Also $\boldsymbol{p}=\left(p_{10}, p_{11}, p_{20}, p_{22}\right)$ and $\boldsymbol{\pi}=\left(\pi_{12}, \pi_{21}, \pi_{02}, \pi_{01}\right)$. The obvious advantage of this simplification is that the model is now more tractable. Since our focus here is fiat money, the fact that commodity money is ruled out will not be a major concern (see Cuadras-Morató and Wright 1997 for a detailed examination of commodity money in a similar context).

We are now in a position to make explicit the value functions for the agents of this economy. For type 1 agents (similarly for type 2) we need to show only $V_{11}$ and $V_{10}$ (we have just argued that commodity money will not play any role here). Let $r$ be the rate of time preference. In flow terms we have

$$
\begin{aligned}
& r V_{11}=-c_{1}+\left[\boldsymbol{\delta}+(1-\boldsymbol{\delta}) \pi_{21}\right] u+(1-\boldsymbol{\delta}) \pi_{01}\left(1-s_{01}\right)\left(V_{10}-V_{11}\right) \\
& r V_{10}=-c_{0}+\left[\delta \pi_{12}\left(1-s_{01}\right)+(1-\delta) \pi_{21}\left(1-s_{02}\right)\right]\left(u+V_{11}-V_{10}\right)
\end{aligned}
$$

Expression (1) represents the value function in flow terms for a type 1 agent holding good 1 at the end of the period. Any agent who ends up holding a consumption good at the end of a period must pay storage cost, $c_{1}$, at the beginning of the next period. In that period the following events may occur. With probability $\delta$, the agent will consume the good he is holding in storage. With probability $(1-\delta) \pi_{21}$, the agent does not want to consume good 1 and goes to the market where she will find an agent holding good 2 and desiring to consume good 1. In this case, trade takes place and the agent consumes good 2. Finally, with probability $(1-\delta) \pi_{01}\left(1-s_{01}\right)$, the agent goes to the market, meets an agent who wants to consume good 1 and holds fiat money and they trade. Any other meeting in the 
market would finish without trade agreement. A similar line of reasoning applies to expression (2). In order to simplify the analysis, we shall adopt the following notation: $\gamma_{1}=\delta+(1-\delta) \pi_{21}$ (similarly for $\left.\gamma_{2}\right)$ and $\gamma_{0}=\delta \pi_{12}\left(1-s_{01}\right)+(1-\delta) \pi_{21}\left(1-s_{02}\right)$. Intuitively, $\gamma_{i}$ is the probability of consumption next period conditional on holding good $i$.

In this particular version of the model, we define an equilibrium (symmetric, steady-state, pure-strategy) as a vector of strategies $\boldsymbol{s}$, such that each $s_{0 i}$ maximizes the payoff of type $i$ agents as described by the value functions $V_{i i}$ and $V_{i 0}$, taking as given the strategies of others. In other words,

DEFINITION. A steady-state, pure-strategy equilibrium is a vector $\boldsymbol{s}$ that satisfies $s_{0 i}=1$ iff $V_{i i}>V_{i 0}$, where the $V$ 's are defined by (1) and (2) and the corresponding expressions for type 2 agents.

The following lemma will be useful to characterize equilibria in the model.

$$
\text { LEMMA 1. For } i=1,2, V_{i i}-V_{i 0}>0 \Leftrightarrow\left(\gamma_{i}-\gamma_{0}\right)>\left(c_{1}-c_{0}\right) / u
$$

Proof. Only solve the system of linear equations (1) and (2) for type 1 agents (and the equivalent expressions for agents of type 2).

The intuition of Lemma 1 is straightforward: good $i$ will be preferred to fiat money if and only if the expected utility of consuming when holding good $i$ minus the storage cost $\left(\gamma_{i} u-c_{l}\right)$ is larger than the expected utility of consuming when holding fiat money minus the corresponding storage cost $\left(\gamma_{0} u-c_{0}\right)$. 
There are three different types of equilibria in this model:

a) Fiat money equilibrium $(\mathrm{M}), \mathbf{s}=(0,0)$, in which all agents accept fiat money.

b) There are two different equilibria in which fiat money is partly accepted, $\mathbf{s}=(1,0)$ and $\mathbf{s}=(0,1)$ (we shall refer to them respectively by $\mathrm{P}$ and $\mathrm{P} ')$. Agents holding good 1 (2) do not accept fiat money in equilibrium $\mathrm{P}$ (P'). Both cases are perfectly symmetric.

c) Barter equilibrium (B), $\mathbf{s}=(1,1)$. No agent will ever accept fiat money. This means that agents always keep their production good until they can consume it or exchange it for something they will consume immediately.

To carry on the analysis we need to solve for the steady-state distribution of inventories of the different agents, $\boldsymbol{p}$. This will be determined by our meeting technology plus the particular trading strategy followed by the agents. In general, we need to solve the following system of equations.

$$
\begin{aligned}
& p_{11}(1-\boldsymbol{\delta})\left(1-p_{11}-p_{22}\right) \boldsymbol{\delta}\left(1-s_{01}\right)=p_{10} \boldsymbol{\delta}(1-\boldsymbol{\delta})\left[p_{11}\left(1-s_{01}\right)+p_{22}\left(1-s_{02}\right)\right] \\
& p_{22}(1-\boldsymbol{\delta})\left(1-p_{11}-p_{22}\right) \boldsymbol{\delta}\left(1-s_{02}\right)=p_{20} \boldsymbol{\delta}(1-\boldsymbol{\delta})\left[p_{11}\left(1-s_{01}\right)+p_{22}\left(1-s_{02}\right)\right]
\end{aligned}
$$

For the different equilibria the results are the following: 
Equilibrium B. The steady-state distribution which corresponds to equilibrium B (that is, trade strategies $\mathbf{s}=(1,1))$ is the following: $p_{11}=\sigma(1-m)=P_{1}, p_{22}=(1-\sigma)(1-m)=P_{2}$, $p_{10}=\sigma m$, and $p_{20}=(1-\sigma) m .^{11}$

Equilibrium M. The steady-state distribution is exactly the same in this case (although the previous note does not apply now).

Equilibrium P. If $1-\sigma \geq m$, the steady-state distribution is $p_{11}=\sigma=P_{1}, p_{22}=1-\sigma-m=$ $P_{2}, p_{10}=0$, and $p_{20}=m$. If $1-\sigma \leq m$, then the distribution is $p_{11}=1-m=P_{1}, p_{22}=0=P_{2}, p_{10}=m$ $(1-\sigma)$, and $p_{20}=1-\sigma^{12}$ (the results for equilibrium $\mathrm{P}^{\prime}$ are symmetric).

Given these results and Lemma 1, we can state now the following Proposition 1.

PROPOSITION 1. a) Equilibrium B exists iff

$$
\frac{c_{1}-c_{0}}{u}<\delta\left[1+\frac{(1-\delta)(1-\sigma)(1-m)}{1-(1-m)[\delta \sigma+(1-\delta)(1-\sigma)]}\right] \text { and }
$$

\footnotetext{
${ }^{11}$ This result depends on the assumption we made about the initial distribution of fiat money among agents. This is because since nobody accepts fiat money in equilibrium B, the agents who were given it in the first place get stuck with it forever. We have assumed that a proportion $m$ of agents of each type was given fiat money in the initial period.

${ }^{12}$ If $\sigma=1-m$, then the steady-state distribution is obviously $p_{11}=\sigma, p_{20}=1-\sigma$, and $p_{10}=p_{22}=0$.
} 


$$
\frac{c_{1}-c_{0}}{u}<(1-\delta)\left[1+\frac{\delta \sigma(1-m)}{1-(1-m)[\delta \sigma+(1-\delta)(1-\sigma)]}\right]
$$

b) Equilibrium $M$ exists iff

$$
\begin{aligned}
& \frac{c_{1}-c_{0}}{u}>\delta\left[1-\frac{(1-\delta) \sigma(1-m)}{1-(1-m)[\delta \sigma+(1-\delta)(1-\sigma)]}\right]>0 \quad \text { and } \\
& \frac{c_{1}-c_{0}}{u}>(1-\delta)\left[1-\frac{\delta(1-\sigma)(1-m)}{1-(1-m)[\delta \sigma+(1-\delta)(1-\sigma)]}\right]>0
\end{aligned}
$$

c) Equilibrium P exists iff

$$
\begin{gathered}
\delta>\frac{c 1-c 0}{u}>(1-\delta)\left[1+\frac{\delta(2 \sigma+m-1)}{1-\delta \sigma-(1-\delta)(1-\sigma-m)}\right]>0 \text { for } 1-\sigma \geq m, \text { and } \\
\delta>\frac{c 1-c 0}{u}>(1-\delta)\left[1+\frac{\delta(1-m)}{1-\delta(1-m)}\right]>0 \text { for } 1-\sigma \leq m
\end{gathered}
$$

(symmetrically for $P^{\prime}$ ).

Proof. The proof is a straightforward application of Lemma 1, given the values for the steady-state distribution of inventories.

The following corollary follows immediately

COROLLARY. Equilibria $P$ and $P$ ' never exist for the same parameter values. 
Proof. Examining conditions of existence of equilibrium P (part c) in Proposition 1) and the symmetric conditions for equilibrium P' (modulating subscripts, which in this case would be changing $1-\delta$ and $1-\sigma$ for $\delta$ and $\sigma$ ), it is easy to check that they cannot be satisfied together.

Proposition 1 makes clear that, independently of the values for the rest of parameters, $c_{1}-c_{0}$ has to be strictly positive and large enough to guarantee existence of equilibria in which fiat money is acceptable. This means that fiat money must have strictly better intrinsic properties than the rest of goods to be valued or, in other words, that $c_{1}-c_{0}$ $\leq 0$ is necessary and sufficient to guarantee that barter equilibrium is unique. This feature of the model differs significantly from previous related literature. Thus, our result is very different from Kiyotaki and Wright (1991) which proves existence of equilibria in which fiat money is used as medium of exchange even when its intrinsic properties are worse than those of the rest of goods. Aiyagari and Wallace (1992) show that, in a two goods model, fiat money is valued only if its storage cost is not larger than that of goods $\left(c_{1}-c_{0} \geq\right.$ 0) (see their Proposition 3.1.). This result is similar to part b) in Proposition 1, although we require that $c_{1}-c_{0}$ be strictly larger than some positive cutoff point which depends on the values of the parameters. In a model with more than two goods, Aiyagari and Wallace (1992) construct examples showing that fiat money may be valued even if it has worse intrinsic properties than the rest of goods. In contrast with this, fiat money can only be valued in our environment if its storability properties are strictly better than those of consumption goods. The reason for this should be intuitively clear: if all goods are equally storable, fiat money will never be preferred to the most valued consumption good, because, at best, they are equally liquid and consumption goods have additional 
consumption value (they can eventually be consumed). Hence, no agent will be able to get the most valued consumption good by holding fiat money, which means that fiat money will necessarily be the least valued object of all and will never be accepted in trade (equilibrium B).

An economy here can be described by a set of parameters $\left(\sigma, \delta, m, c_{1}, c_{0}, u\right)$. Proposition 1 allows us to identify the equilibria for any such economy. In order to illustrate this, we present several figures representing the existing equilibria in the parameter space $(\delta, \sigma)$, given particular values for $m$ and $\left(c_{1}-c_{0}\right) / u$ (Figures 1-9). Notice that the figures show points for which equilibria $\mathrm{P}, \mathrm{M}$, and $\mathrm{B}$ exist and omit the representation of equilibrium P'. Nothing is lost with this, because equilibrium P' is perfectly symmetric of $\mathrm{P}$ along the $45^{\circ}$ line (and we simply chose not to show it in the figures).

Several characteristics of the figures are worth a comment. First, multiplicity of equilibria is a pervasive feature of search models of money. There are large areas of the parameter space $(\delta, \sigma)$ for which there are two or three equilibria. The explanation for this is commonplace in this type of models: money is a social convention and what appears as medium of exchange not only depends on the fundamental parameters of the economy, but also on the way agents coordinate on a particular equilibrium. In general, equilibria cannot be Pareto ranked, although some interesting results will be presented below concerning this matter. Second, our definition of equilibrium included only pure strategies. From the figures, it is clear that there are some areas in which no such 
equilibrium exists. ${ }^{13}$ We can also evaluate the values of the parameters for which different equilibria exist. Equilibrium P exist for high enough values of $\delta$, whatever the values of $m$ and $\left(c_{1}-c_{0}\right) / u$. Rather intuitively, equilibrium B is more likely to exist for low values of $\left(c_{1}-c_{0}\right) / u$, while equilibrium $\mathrm{M}$ is more likely to appear for relatively high values. Equilibrium $\mathrm{M}$ and $\mathrm{B}$ do not appear for extreme values of $\delta(\delta \rightarrow 0,1)$. The intuition for this is straightforward: if $\delta \rightarrow 1$, good 1 is too valuable to be exchanged for fiat money, so equilibrium $\mathrm{M}$ does not exist; also, good 2 is worth very little as asset and it is costly to hold as well, so agents will accept fiat money to save on storage costs, and equilibrium B will not exist. Obviously, for very close to zero storage costs the only equilibria that will eventually exist is B. This is because storage costs or some other intrinsic property of goods are crucial here: without them we would not get valuable fiat money, just as in Cuadras-Morató and Wright (1997).

Next issue we want to discuss is welfare. We take the following measure of welfare for individuals of type $i: W_{i}=\left(1 / \sigma_{i}\right)\left(p_{i i} V_{i i}+p_{i 0} V_{i 0}\right)$. This is a measure which computes welfare of individuals before the uncertainty derived from the initial random distribution of inventories is resolved (see Kiyotaki and Wright 1993). Take for instance the case of equilibrium B. Agents who are given fiat money at the beginning, stay with it indefinitely and, then, their lifetime utility (computed after this source of uncertainty is resolved) would be zero. We consider ex-ante welfare, that is, before the initial distribution of inventories take place and all agents have the same probability of getting

\footnotetext{
${ }^{13}$ Our conjecture is that these areas correspond to values of the parameters for which only mixed strategy equilibria exist. Unfortunately, including mixed strategies into the model makes it rather nontractable, even for solving numerical examples.
} 
fiat money or their production good. The main result we present about welfare is summarized in the following proposition.

PROPOSITION 2. $V_{i j}^{M}>V_{i j}^{B}, \forall j=i, 0 .^{14}$

Proof. Substituting for $\boldsymbol{s}$ and $\boldsymbol{\pi}$ in the $V_{i j}$ corresponding to equilibria $\mathrm{M}$ and $\mathrm{P}$ we can show that $V_{i 0}^{M}>V_{i 0}^{B}=0$ and $V_{i i}^{M}>V_{i i}^{B}$ for the values of parameters for which $\mathrm{M}$ and B exist together. The proof involves simple algebraic manipulations.

An immediate corollary of Proposition 2 is $W_{i}^{M}>W_{i}^{B}$. Proposition 2 guarantees that fiat money equilibrium is efficient when compared with barter equilibrium for all the values of the parameters for which they coexist. Money holders are obviously better off in a monetary equilibrium in which fiat money takes on value than in a barter equilibrium where fiat money is valueless. At the same time, consumption good holders are better off in a fiat money equilibrium, since now there is an alternative asset, fiat money, which is less costly to store and, at the same time, it is perfectly accepted by all agents (although it cannot be consumed).

The structure of the model makes very difficult to derive analytically other welfare results. Nevertheless, we will state results based on numerical examples that analyze different economies characterized by different values of the parameters. In particular, we are interested in the following result: for all the different values of the

\footnotetext{
${ }^{14}$ Superscripts M (B or P) refer to fully accepted fiat money, barter, and partly accepted fiat money equilibria respectively.
} 
parameters we tried, $W_{i}^{P}>W_{i}^{B}$ (we proved analytically this result for agent of type 1 , but could not do it for agents of type 2). This result seems important because it means that partial use of fiat money (in equilibrium $\mathrm{P}$ only agents of type 2 accept fiat money) is also welfare enhancing.

Summing up, we have presented two main results in this section. First, it has been shown that for a large set of economies there exist an equilibrium in which fiat money is valued by everyone; and, second, we have proved that this fiat money equilibrium is welfare enhancing with respect to barter. We have shown these results in the context of a simple model with only two consumption goods $(n=2)$, in which there are not problems of double coincidence of wants and, consequently, money does not play any role as medium of exchange. In fact, the only role of money in the model is as store of value. We have proved that, in order to be valued, fiat money has to be better storable than the rest of goods. Of course, questions may remain about the robustness of these results in a more general model in which $n>2$. Although we do not present a formal proof, it should be clear that the argument holds in a general model since, with many consumption goods, nothing really changes in the nature of the model. Although the potential of fiat money as medium of exchange (in other words, its liquidity value) might be larger because of the problem of double coincidence of wants, this does not offset its null consumption value. Consequently, fiat money can only take on value if its intrinsic properties are better than those of the rest of goods are. In fact, this is what we have proved elsewhere in the context of a model in which $n=3$ (Cuadras-Morató and Wright 1997, Proposition 3). In other words, the comparative advantage of fiat money in this type of models is its potential role as store of value and this is independent of the value of $n$. 


\section{Government Transaction Policy and Fiat Money}

As we have argued above, it might be necessary to study the way government gets involved in the process of exchange in order to study the emergence and use of money in the economy. Government is regarded as an important agent whose behavior could influence other agents' trading strategies. To allow for these considerations, the model is now modified in a basic aspect, so we can analyze the role of government transaction policy in the determination of the medium of exchange. In order to keep the analysis simple, we also suppress storage costs (which are not central to our analysis now), so that now all commodities are storable at no cost. ${ }^{15}$

We now introduce the role of government transaction policy into the problem of the determination of the medium of exchange. In order to do this, we need to formalize the existence of a new set of agents, which will be named government agents (our modeling of the government follows Aiyagari and Wallace (1997); see also Li and Wright 1998 and Ritter 1995). Agents in the economy will be of two different types: a proportion $G$ of agents will be government agents, while the rest $1-G$ will be private agents. Private agents have exactly the same characteristics of the agents we have described in the previous sections: they produce according to their respective specialization and they have

\footnotetext{
${ }^{15}$ As we shall see below, the results of the following sections hold as long as storage costs associated to fiat money are not too low compared to the rest of commodities.
} 
a stochastic structure of preferences for all goods. The new ingredient now is the government sector. These agents do not produce anything and they do not consume either. They only go to the market, meet other agents and trade (we can think about them as if they were vending machines) according to some predetermined rule, which we shall call the government transaction policy. In this economy, the government adopts the following transaction policy: always accept fiat money, always give up fiat money, and exchange a consumption good for another consumption good with probability $\theta^{16}$ The parameter $\theta$ is measuring the degree with which the government is favoring the emergence of fiat money as the medium of exchange. The lower the value of $\theta$, the more favored is the emergence of fiat money as medium of exchange by the government policy. If $\theta=1$, then holding fiat money does not make easier to get a consumption good held by a government agent when compared to holding any other commodity (that is, it reduces the exchange value of fiat money).

The rest of the elements of the model basically do not change. There is an amount $m$ of fiat money (we assume $G>m$ ). The strategic decision to be taken by private agents (government agents do not actually take any decision) is identical and again they are just asked to rank all objects as before. Also the way the value functions are defined is exactly the same, with the addition of the possibility of now meeting government agents. The definition of equilibrium also holds with no significant changes.

We must introduce now some notation related with the distribution of inventories, which has slightly changed. As before, let $p_{i j}$ be the measure of traders of type $i$ $(i=1,2, \ldots, n)$ who have $\operatorname{good} j(j=0,1,2, \ldots, n)$ in inventory and let $p_{G j}$ be the measure of

${ }^{16} \mathrm{See} \mathrm{Li}$ and Wright (1998) for a detailed analysis of more general transaction policies. 
government agents who happen to have in inventory good $j$. Let $\boldsymbol{p}=\left(\ldots, p_{i j}, p_{G j}, \ldots\right)$ be the distribution vector. Obviously, the summation of all elements of vector $\boldsymbol{p}$ is equal to one. Moreover, $\sum_{j} p_{G j}=G, \sum_{j} p_{i j}=\sigma_{i}(1-G)$. Let $P_{j}=\sum_{j} p_{i j}$ and $\sum_{j} P_{j}=1-G$. The measure of agents in the economy who hold fiat money is now $m=P_{0}+p_{G 0}$. Every period of time, a proportion $N\left(N=1-\sum_{i} \delta_{i} P_{i}\right)$ of agents go to the market and are randomly matched with other agents who are in the same situation. As before, any agent who goes to the market will meet a trader who holds good $j$ and desires to consume good $i$ with probability $\left.\pi_{j i}=\left(P_{j} \delta_{i}\right) / N\right)$. Similarly, any agent going to the market will meet with probability $\alpha_{j}=p_{G j} / N$ a government agent holding good $j$. It is easy to see that $\sum_{j} \alpha_{j}+\sum_{i} \sum_{j} \pi_{j i}=1$. The rest is identical to the previous version of the model: given $\boldsymbol{s}$, the distribution $\boldsymbol{p}$ evolves according to some law of motion $\boldsymbol{p}^{\prime}=f(\boldsymbol{p} ; \boldsymbol{s})$. A steady-state distribution is a solution to $\boldsymbol{p}=f(\boldsymbol{p} ; \boldsymbol{s})$. Once we know $\boldsymbol{p}$, it is easy to determine the steady-state value of $\boldsymbol{\pi}=\left(\ldots, \pi_{i j}, \alpha_{j}, \ldots\right)$ as a function of $\boldsymbol{s}$ (given the rest of the parameters of the model, $\boldsymbol{\delta}, \boldsymbol{\sigma}, G, \boldsymbol{\theta}$, and $m$ ). As usual in this kind of models, we shall assume that agents have rational expectations about meeting probabilities.

\section{An Example: Government Transaction Policy with Two Consumption}

\section{Goods and Fiat Money}

We take $n=2$ to simplify the model and make it more tractable. As in the previous model, agents will never have to choose between consumption goods. There are only two goods here, so whenever an agent is in the market with a consumption good, this can be only because she wants to consume the other good. This implies that she will accept it whenever it is offered to her. This means that we can summarize trading strategies of 
agents with a vector $s=\left(s_{01}, s_{02}\right)$, just as before. Nevertheless, it is now possible that an agent of type 1 (2) is holding good 2 (1) at some point. The reason for this is that now she could get it from a government agent who is always willing to exchange it for fiat money. Let us be more explicit about the derivation of the value functions in the new version of the model. The payoff functions, in flow terms, for a trader of type 1 (similarly for type 2 ) can be written as follows:

$$
\begin{gathered}
r V_{11}=\delta u+(1-\delta)\left[\left(\pi_{21}+\alpha_{2} \theta\right) u+\left(\pi_{01}+\alpha_{0}\right)\left(1-s_{01}\right)\left(V_{10}-V_{11}\right)\right] \\
r V_{12}=(1-\delta)\left(u+V_{11}-V_{12}\right)+\delta\left[\left(\pi_{12}+\alpha_{1} \theta\right)\left(u+V_{11}-V_{12}\right)+\pi_{02}\left(1-S_{02}\right)\left(V_{10}-V_{12}\right)\right] \\
r V_{10}=\delta\left\{\left[\pi_{12}\left(1-s_{01}\right)+\alpha_{1}\right]\left(u+V_{11}-V_{10}\right)+\alpha_{2 S 02}\left(V_{12}-V_{10}\right)\right\}+ \\
+(1-\delta)\left\{\left[\pi_{21}\left(1-S_{02}\right)+\alpha_{2}\right]\left(u+V_{11}-V_{10}\right)+\alpha_{1 S 01}\left(V_{11}-V_{10}\right)\right\}
\end{gathered}
$$

Expressions (5) and (7) are very similar to expressions (1) and (2). There are a few obvious differences worth commenting. First, storage costs have disappeared from the picture now. Second, we have the expression (6) for $V_{12}$, which was not present before. Third, traders can now meet government agents who are holding good $j$ with probability $\alpha_{j}(j=0,1,2)$.

Given the definition of equilibrium, analogous to the previous section, there are also the same type of equilibria in the model: M, B, and P (and its symmetric P').

Although the model is similar to the one we analyzed before, it is rather more difficult to solve. The difficult bit is, above all, computing the steady-state distribution of inventories, which has now become more complicated with the presence of government agents. Due to this, it will be impossible to get general analytical results. Nevertheless, 
we shall present a few findings, which will be useful to solve the model numerically and also will help to understand important characteristics of this economy. First, the following Lemma 2 presents necessary and sufficient conditions for existence of the different equilibria.

\section{LEMMA 2.}

$$
\begin{aligned}
& V_{i 0}>V_{i 1} \text { iff }\left(\boldsymbol{\gamma}_{0}-\boldsymbol{\gamma}_{1}\right)\left(\boldsymbol{\varepsilon}_{1}+\boldsymbol{\gamma}_{2}+r\right)+\left(\boldsymbol{\gamma}_{2}-\boldsymbol{\gamma}_{1}\right) \eta_{l}>0 \text { (8) } \\
& V_{i 0}>V_{i 2} \text { iff }\left(\boldsymbol{\gamma}_{0}-\boldsymbol{\gamma}_{2}\right)\left(\boldsymbol{\varepsilon}_{2}+\boldsymbol{\gamma}_{1}+\boldsymbol{r}\right)+\left(\boldsymbol{\gamma}_{1}-\boldsymbol{\gamma}_{2}\right) \eta_{2}>0 \text { (9) where } \\
& \varepsilon_{l}=\delta\left(1-S_{02}\right)\left(\pi_{02}+\alpha_{0}\right), \quad \eta_{l}=\delta \alpha_{2} S_{02}, \quad \gamma_{1}=\delta+(1-\delta)\left(\pi_{2 l}+\alpha_{2} \theta\right), \\
& \varepsilon_{2}=(1-\delta)\left(1-s_{01}\right)\left(\pi_{01}+\alpha_{0}\right), \quad \eta_{2}=(1-\delta) \alpha_{1 S 01}, \quad \gamma_{2}=1-\delta+\delta\left(\pi_{12}+\alpha_{1} \boldsymbol{\theta}\right), \quad \text { and } \\
& \gamma_{0}=\delta\left[\pi_{12}\left(1-s_{01}\right)+\alpha_{1}\right]+(1-\delta)\left[\pi_{21}\left(1-s_{02}\right)+\alpha_{2}\right]
\end{aligned}
$$

Proof. Take the system of linear equations (5), (6), and (7). Solve for $V_{l j}(\mathrm{j}=0,1,2)$ and check the conditions under which $V_{10}>V_{1 i}(\mathrm{i}=1,2)$. The argument would be identical for traders of type 2).

We can now prove the following Proposition 3.

PROPOSITION 3. Equilibrium $M$ does not exist in this economy.

Proof. Substituting for the value of $s_{0 i}=0$ in (8) and (9) gives us that equilibrium M will exist iff $\gamma_{0}>\gamma_{i}$. Substituting in the expressions for $\gamma_{s}$ in Lemma 2 we have the following two conditions:

$$
(1-\delta) \alpha_{2}(1-\theta)>\delta\left(1-\pi_{12}-\alpha_{1}\right)
$$




$$
\delta \alpha_{1}(1-\theta)>(1-\delta)\left(1-\pi_{21}-\alpha_{2}\right)
$$

From (10) and (11):

$$
\begin{aligned}
& \frac{1-\delta}{\delta}>\frac{1-\pi_{12}-\alpha_{1}}{\alpha_{2}(1-\theta)} \\
& \frac{1-\delta}{\delta}<\frac{\alpha_{1}(1-\theta)}{1-\pi_{21}-\alpha_{2}}
\end{aligned}
$$

So,

$$
\frac{\alpha_{1}(1-\theta)}{1-\pi_{21}-\alpha_{2}}>\frac{1-\pi_{12}-\alpha_{1}}{\alpha_{2}(1-\theta)}
$$

Operating this expression and substituting for $\pi$ 's and $\alpha$ 's corresponding to equilibrium $\mathrm{M}$, one easily derives the following contradiction:

$$
m\left(1-\delta P_{1}-P_{2}\right)+p_{G 2} P_{1}(1-\delta)+\delta P_{2}\left(1-\delta P_{1}-P_{2}-p_{G 2}\right)<0
$$

Hence, equilibrium $\mathrm{M}$ does not exist in this model (for any values of the parameters).

The intuition behind this result is rather simple. The value of holding any commodity (in terms of the probability of consumption) can be deconstructed in two different components: consumption value (any commodity, except fiat money, might eventually be consumed); and liquidity value (commodities might be exchanged in the 
market for other commodities that might be consumed). Fiat money has zero consumption value and its liquidity value is equal to $\delta\left(\pi_{21}+\alpha_{1}\right)+(1-\delta)\left(\pi_{12}+\alpha_{2}\right)$, given that $s_{0 i}=0$. Good 1 has consumption value equal to $\delta$ and liquidity value $(1-\delta)\left(\pi_{12}+\alpha_{2} \theta\right)$. Similarly, good 2 has consumption value $1-\delta$ and liquidity value $\delta\left(\pi_{21}+\alpha_{1} \theta\right)$. Note that $\theta=0$ means that fiat money has relatively large liquidity value with respect to consumption goods. Even in this case, it is easy to see that fiat money cannot have larger value than all consumption goods. In fact, fiat money always has greater liquidity value than the rest of goods, but has never greater total value than all of them. This is because always at least one good has consumption value large enough to compensate for its lower liquidity value relative to fiat money. This is what Proposition 3 shows: fiat money cannot be better asset than all consumption goods in the economy and, then, equilibrium $\mathrm{M}$ does not exist. This is in contrast with the result in Cuadras-Morató and Wright (1997) (see Proposition 3 in that paper) in which fiat money does not have any value as medium of exchange. There, fiat money is always the least valued object. Proposition 3 above states that fiat money cannot be the most valued object, although there are still many instances in which fiat money will be used by a vast majority of agents. Fiat money might have better liquidity value than all goods, but cannot be better valued than the most valued consumption good(s).

Proposition 3 is substantially different from previous recent results from models that analyze government transaction policy. Thus, Aiyagari and Wallace (1997) (see Proposition 1 in that paper) and Li and Wright (1998) (see Proposition 1 in that paper) both identify general conditions under which there exist a monetary equilibrium (unique or not) in which all agents in the economy use fiat money as medium of exchange. This is precisely what we cannot get in our present set up. In the model we analyze here, we do not have such equilibrium. There are equilibria in which fiat money is used rather 
generally, but never by all agents. In particular, agents holding the best-valued consumption good (gold) will never accept fiat money.

The following lines present other results of the model. First, Proposition 4 displays results about existence of equilibria $\mathrm{B}$ and $\mathrm{P}\left(\mathrm{P}^{\prime}\right)$ for extreme values of some parameters.

\section{PROPOSITION 4.}

a) If $G \rightarrow 0$, equilibrium $B$ exists and is unique;

b) If $\delta \rightarrow 0(1)$, equilibrium $P\left(P^{\prime}\right)$ does not exist;

c) $\theta \rightarrow 0$ is sufficient condition for nonexistence of equilibria $P$ (and by symmetry $\left.P^{\prime}\right)$;

Proof. The proof is simple and proceeds by examining the equilibrium conditions (8) and (9) from Lemma 2. Analyzing equilibrium B, conditions (8) and (9) are the following:

$$
\begin{aligned}
& \left\{\delta\left(\alpha_{1}-1\right)+(1-\delta)\left[\alpha_{2}(1-\theta)-\pi_{21}\right]\right\}\left[1-\delta+\delta\left(\pi_{12}+\alpha_{1} \theta\right)+r\right]+ \\
& +\delta \alpha_{2}\left[(1-\delta)\left(1-\pi_{21}-\alpha_{2} \theta\right)-\delta\left(1-\pi_{12}-\alpha_{1} \theta\right)\right]<0 \quad(12) \text { and } \\
& \left\{(1-\delta)\left(\alpha_{2}-1\right)+\delta\left[\alpha_{1}(1-\theta)-\pi_{12}\right]\right\}\left[\delta+(1-\delta)\left(\pi_{21}+\alpha_{2} \theta\right)+r\right]+ \\
& +(1-\delta) \alpha_{1}\left[\delta\left(1-\pi_{12}-\alpha_{1} \theta\right)-(1-\delta)\left(1-\pi_{21}-\alpha_{2} \theta\right)\right]<0
\end{aligned}
$$

Analyzing equilibrium P (by symmetry of the model, the analysis of equilibrium P' is similar) conditions (8) and (9) look like 


$$
\begin{gathered}
(1-\delta) \alpha_{2}(1-\theta)-\delta\left(1-\alpha_{1}\right)<0 \quad \text { (14) and } \\
\left\{\delta\left[\alpha_{1}(1-\theta)-\pi_{12}\right]-(1-\delta)\left(1-\alpha_{2}-\pi_{21}\right)\right\}\left[\delta+(1-\delta)\left(\pi_{21}+\alpha_{2} \theta\right)+r\right]+ \\
+(1-\delta) \alpha_{1}\left[\delta\left(1-\pi_{12}-\boldsymbol{\alpha} \boldsymbol{\theta}\right)-(1-\delta)\left(1-\pi_{21}-\alpha_{2} \theta\right)\right]>0
\end{gathered}
$$

a) Suppose $G \rightarrow 0$, then $\alpha_{1}, \alpha_{2} \rightarrow 0$ and (12) and (13) always hold, so equilibrium B always exists. Also, (15) will never hold, so equilibrium P (and P', by symmetry) does not exist.

b) Suppose $\delta \rightarrow 0$, then (14) will never hold and, so, $\mathrm{P}$ does not exist. Symmetrically, if $\delta \rightarrow 1$, P' does not exist.

c) Also if $\theta \rightarrow 0$, then $p_{G l} \rightarrow 0^{17}$, so $\alpha_{1} \rightarrow 0$ and again (15) will never hold, so equilibrium $\mathrm{P}\left(\mathrm{P}^{\prime}\right)$ does not exist.

Proposition 4 reflects the analysis of the model for some extreme values of the parameters for which the model becomes tractable enough. The intuition for result a) is fairly easy to understand. If the number of government agents is very small, no matter which are the parameters of the economy and the government transaction policy, fiat money loses its appeal and agents in the economy will rank fiat money as the least valued asset (just like in the commodity money model of Cuadras-Morató and Wright 1997). Part b) can also easily be justified. If $\delta$ is very big, then good 2 is very unattractive and, hence,

\footnotetext{
${ }^{17}$ In order to get this particular result, we need to solve for the steady-state distribution of inventories. In general, this involves solving a system of nonlinear equations the details of which can be found in the appendix.
} 
is never preferred to fiat money which means that equilibrium P' cannot exist. Finally, part c) is a little bit more complicated to justify intuitively. Basically what happens is the following. If the government policy does not allow government agents to exchange good 2 for good 1 then, in steady state, government agents will hold good 1 with probability zero, because they cannot get it from holding fiat money and exchanging it (no agent will prefer fiat money to good 1 in equilibrium $\mathrm{P}$ ). The consequence of this is that the exchange value of fiat money is zero even for type 2 agents, because it would only be useful for agents of type 2 to hold fiat money if they could meet government agents who held good 1.

Solving this new model is more complicated than solving the one in the previous section and this means that we cannot derive general analytical results similar to Proposition 1. The main difficulty here is solving for a slightly more complicated steadystate inventory distribution, which now includes government agents. ${ }^{18}$ In the Appendix, we present the system of nonlinear equations we need to solve to find the steady-state inventory distribution for different economies described by a different set of parameters $(\delta, \sigma, m, G, \theta, r)$. In order to illustrate the workings of the model we recur to numerical methods to solve it for a number of different economies and present equilibria $\mathrm{B}$ and $\mathrm{P}$ ' (by the symmetry properties of the model we do not need to present equilibrium $\mathrm{P}$ ) in different graphs. Basically what we do is the following: first, we take values of the parameters $m, G, \theta$ and $r$; second, we compute the steady-state inventory distribution and check for existence of equilibria (applying Lemma 2) for all points on a grid of the set $\Theta=\{\delta, \sigma\}$; and third, we represent the results in the parameter space $\{\delta, \sigma\}$. Figures $10-14$ are examples which illustrate the results of this exercise. As in the previous model, we

\footnotetext{
${ }^{18}$ This complication will make impossible to present relevant welfare results. We could only get some numerical results for the special case in which $n=2$.
} 
have multiple equilibria for large areas of the space of parameters (e.g. Figure 13). Also, there are values of the parameters for which there does not exist pure strategy equilibrium (e.g. Figure 14). It is possible now to find examples of mixed strategy equilibria that rather naturally fill those gaps. For instance, for $m=0.05, G=0.3, \theta=0.2, r=0.00001$, $\delta=0.13$, and $\sigma=0.07$ (see Figure 14) there does not exist any pure strategy equilibrium, but it can be shown that $s=(0.404,1)$ constitutes a mixed strategy equilibrium.

The nonexistence result contained in Proposition 3 is the most important conclusion of this section so far. This main result could be rephrased in the following way: fiat money cannot be the best valued (most accepted) object. It could be accepted in exchange by almost all commodity holders in the economy, but never by all. There is at least one good (gold), which is the most valued of all consumption goods, which dominates fiat money. It is not that fiat money is not valued in those models (as it happens, for instance, in Cuadras-Morató and Wright (1997)): we only state that it cannot be the most valued object in the economy. In this respect, an important question arises concerning the robustness of this conclusion to the general case in which $n>2$ : does this result follow for a model with more than two consumption goods? In a sense, one could think that the more goods there exist, the lower would be the consumption value of each single one and, hence, it would be possible to have fiat money better accepted than any other good (we could think of a case like $n>2$ where $\delta_{i}=1 / n$, for instance). Also, with more than two goods, a problem of double coincidence of wants arise, so that fiat money might now be valued as medium of exchange. This basically means that fiat money may be more valued than most goods. Our result states, however, that fiat money is not more valued than all goods. It is important to check for this, otherwise it would mean that the result in Proposition 3 does not survive small changes in the environment. Unfortunately, 
we were not able to come up with a general proof (the model is far too complicated to do this). We can, however, present a proof of exactly the same result in an economy with $n>2$ and $\delta_{i}=1 / n$ (all goods have exactly the same "demand"). Given the way the model works, this structure of preferences is the one that could give rise most easily to universally accepted fiat money since no consumption good has a larger "demand" than the rest, which makes it most acceptable. In this sense, if this nonexistence result is true for this particular case, it should be true for any other case.

\section{A General Model of Goods with the Same Demand $\left(\delta_{i}=\delta\right)$}

The economy is just as before and can be characterized by parameters $\delta=\left\{\boldsymbol{\delta}_{l}\right.$, $\left.\delta_{2}, \ldots \delta_{n}\right\}, \boldsymbol{\sigma}=\left\{\sigma_{1}, \sigma_{2}, \ldots, \sigma_{n}\right\}, m, G, \boldsymbol{\theta}$, and $r$. Equilibrium $\mathrm{M}$ (an equilibrium in which fiat money is the most valued asset) would not exist in none of the economies we tried numerically for $n=3$. We turn directly to the proposition which shows the result for $n>2$ in the particular case for which $\delta_{i}=1 / n .^{19}$

PROPOSITION 5. Equilibrium $M$ does not exist for an economy in which $n>2$ and $\delta_{i}=1 / n$.

\footnotetext{
${ }^{19}$ In terms of making more likely the emergence of fiat money as the most valued asset, choosing the values of the parameters $\delta_{i}=1 / \mathrm{n}$ is the best choice, since does not give any extra consumption value to any commodity, which then would appear as the best asset for that reason.
} 
Proof. Here we sketch the proof of the proposition, the logic of which is similar to the proof of Proposition 3. We focus on showing nonexistence of equilibrium with fiat money as the best valued asset of all in an economy in which the other assets are arbitrarily ranked as follows: the best consumption good is 1 , the second 2, the third is 3 , etc. That is, trade strategies are $s=\left(s_{01}, s_{12}, s_{23}, \ldots\right)=\left(s_{01}, 0,0, \ldots\right)$. A similar system of equations to (5), (6), and (7) (a system of $n+1$ equations now, of course) would ensure that $V_{i 0}>V_{i 1}$ iff $\gamma_{0}>\gamma_{1}$ (a similar result to Lemmas 1 and 2). This is equivalent to

$$
\sum_{i=1}^{n}\left[\frac{\delta_{i}\left(1-\delta_{i}\right) P_{i}}{N}+\frac{\delta_{i} p_{G i}}{N}\right]-\boldsymbol{\delta}_{1}-\sum_{j=2}^{n}\left[\frac{\delta_{j}\left(1-\delta_{j}\right) P_{j}}{N}+\frac{\delta_{j} p_{G j}}{N}\right]>0
$$

Substituting for $\delta_{i}=\boldsymbol{\delta}(=1 / n)$ and operating, we get the following contradiction

$$
\left(G+P_{1}-1\right)(1-\delta)+\boldsymbol{\theta}\left(p_{G 0}+p_{G 1}-G\right)>p_{G 0}
$$

which clearly cannot be. So equilibrium $\mathrm{M}$ does not exist.

\section{Summary and Concluding Remarks}

The exercise carried out in this paper can be summarized as follows. We start from a commodity money model without any role for fiat money (based on CuadrasMorató and Wright 1997). We introduce fiat money in the model using two different approaches. First, we model fiat money with different intrinsic properties (storability) than consumption goods. We show that in this particular context, fiat money can only take on value if it has better intrinsic properties than the rest of goods. This result differs from 
previous search theoretical literature on money that emphasized the possibility of existence of equilibria in which commodities that do not have the most desirable properties circulate as general media of exchange. Second, we introduce government transaction policy following Aiyagari and Wallace (1997). The main result now is that there does not exist an equilibrium in which fiat money dominates the rest of all commodities as circulating media of exchange. Pieces of paper may appear as money if government policy favors it, but they will never be more acceptable than the most valued goods (gold, for instance). This result is different from other papers that introduce the subject of government transaction policy on money (Aiyagari and Wallace 1997 and Li and Wright 1998) and generally find that there are equilibria in which fiat money is the only media of exchange.

We believe these results can tell us a few things about early episodes of unconvertible fiat money. First, there is not unconvertible fiat money unless there is some sort of government intervention. Our first model predicts that fiat money would only appear if it had better intrinsic properties than the rest of commodities. It is very doubtful that this would be so with private firms issuing it. In that case, uncertainty about its value would always make unconvertible paper a less interesting medium of exchange. Government policy might give money a better chance to circulate for transaction purposes, and this is what we show in the second part of our paper. Nevertheless, fiat money cannot dominate gold or other commodities that are generally accepted as money. What we have are economies in which fiat money circulates along with other commodities that are also used as media of exchange, without ever dominate them. Arguably, this is what happened in the early episodes of fiat money we mentioned in the paper. 
There are several extensions of this model worthwhile mentioning here. First, the introduction of bargaining over the terms of trade which would allow us to make prices endogenous and discuss more explicitly about topics such as inflation. Second, the introduction of several currencies with different properties will give rise to the issues of currency substitution and rates of exchange. Finally, we have studied only a very limited type of government policy. One could think about different policies and the way they affect the conclusions we have drawn in this paper.

\section{Appendix}

Given the set of parameters $(\delta, \sigma, m, G, \boldsymbol{\theta}, r)$, the matching technology of traders, the government transaction policy, and the trading strategies followed by traders, $s$, the steady-state distribution of holdings is determined solving the following system of nonlinear equations.

For agents of type 1,

$$
\begin{gathered}
p_{12}\left[1-\delta+\delta\left(\pi_{12}+\alpha_{1} \theta\right)\right]+p_{10}\left\{\delta\left[\pi_{12}\left(1-s_{01}\right)+\alpha_{1}\right]+\right. \\
\left.(1-\delta)\left[\pi_{21}\left(1-s_{02}\right)+\alpha_{2}\right]+(1-\delta) \alpha_{1 S 01}\right\}=p_{11} \delta_{2}\left(1-s_{01}\right)\left(\pi_{01}+\alpha_{0}\right) \\
p_{10} \delta_{1} \alpha_{2 S 02}=p_{12}\left\{1-\delta+\delta\left[\pi_{12}+\alpha \boldsymbol{\alpha}+\left(1-s_{02}\right)\left(\pi_{02}+\boldsymbol{\alpha}_{0}\right)\right]\right\}
\end{gathered}
$$

Modulating appropriately the subscripts, we would have similar equations for agents of type 2. Finally, for government agents, 
$\boldsymbol{\pi}_{12}\left[p_{G 0}\left(1-S_{01}\right)+p_{G 2} \boldsymbol{\theta}\right]=p_{G 1}\left(\boldsymbol{\pi}_{2} \boldsymbol{\theta}+\boldsymbol{\pi}_{01}+\boldsymbol{\pi}_{02 S 01}\right)$

$\boldsymbol{\pi}_{21}\left[p_{G 0}\left(1-S_{02}\right)+p_{G} \boldsymbol{\theta}\right]=p_{G 2}\left(\boldsymbol{\pi}_{12} \boldsymbol{\theta}+\boldsymbol{\pi}_{02}+\boldsymbol{\pi}_{01 S 02}\right)$ 


\section{References}

Aiyagari, S. Rao and Neil Wallace (1992) "Fiat money in the Kiyotaki-Wright model" Economic Theory, 2, 447-464

Aiyagari, S. Rao and Neil Wallace (1997) "Government transaction policy, the medium of exchange, and welfare", Journal of Economic Theory, 74, 1-18

Chown, John F. (1994) A History of Money London, Routledge

Cuadras-Morató, Xavier and Randall Wright (1997) "Money as a medium of exchange when goods vary by supply and demand" Macroeconomic Dynamics, 1, 680700

Friedman, Milton and Anna J. Schwartz (1986) "Has Government any role in Journal of Monetary Economics, 17, 37-62

Jevons, Stanley (1875) Money and the Mechanism of Exchange London, King

Kiyotaki, Nobuhiro and Randall Wright (1989) "On money as a medium of exchange" Journal of Political Economy, 97, 927-954

Kiyotaki, Nobuhiro and Randall Wright (1991) “A contribution to the pure theory Journal of Economic Theory, 53, 215-235 
Li, Yiting and Randall Wright (1998) "Government transaction policy, media of Journal of Economic Theory, 81, 290-313

Menger, Karl (1892) “On the origin of money” Economic Journal, 2, 239-255

Ritter, Joseph (1995) "The transition from barter to fiat money" American Economic Review, 85, 134-149

Shi, Shouyong (1995) "Money and prices: a model of search and bargaining" Journal of Economic Theory, 67, 467-496

The Economist (1999) "Paper gains" Volume 353, n. 8151, $31^{\text {st }}$ December

Trejos, Alberto and Randall Wright (1995) "Search, bargaining, money and Journal of Political Economy, 103, 118-141 
Figure 1

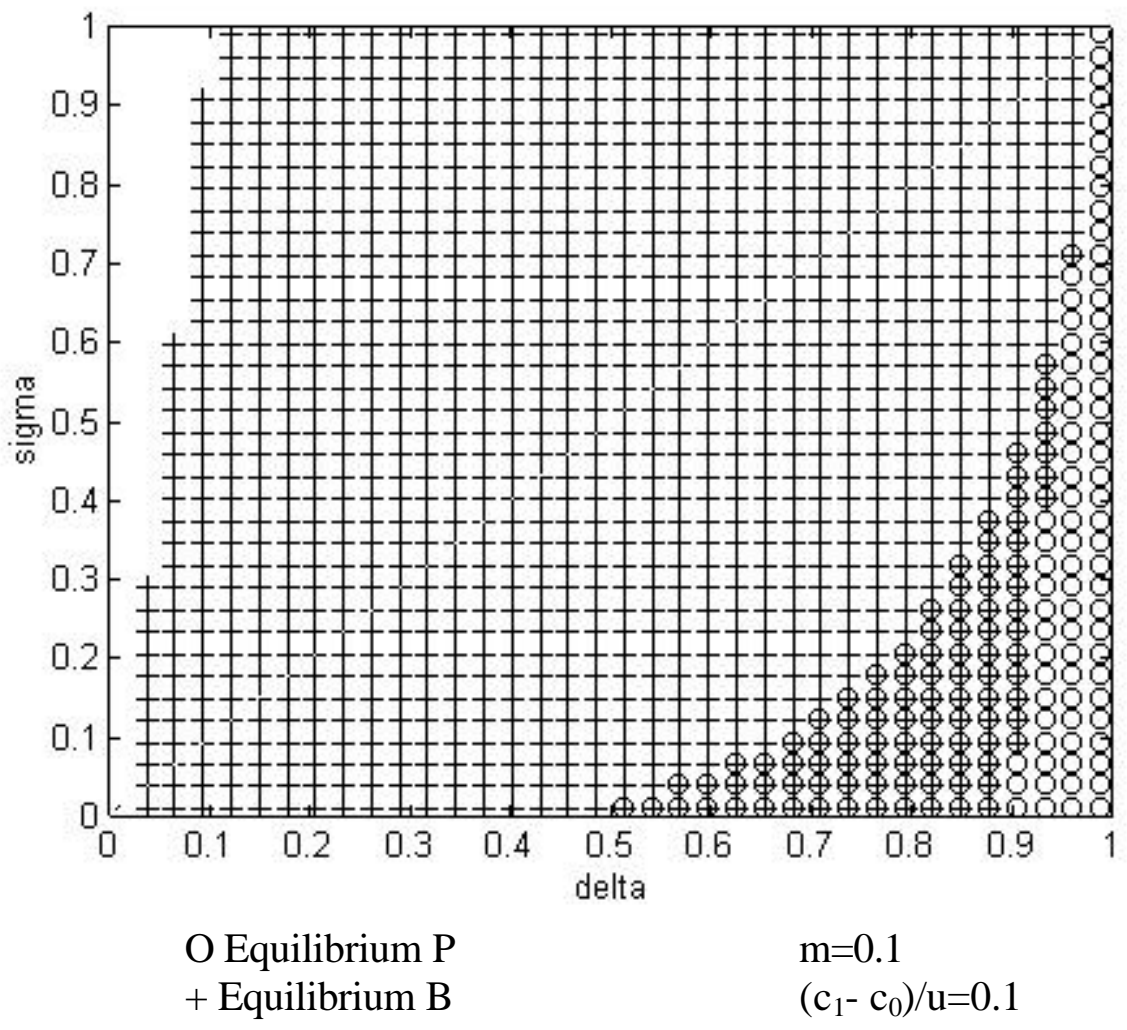


Figure 2

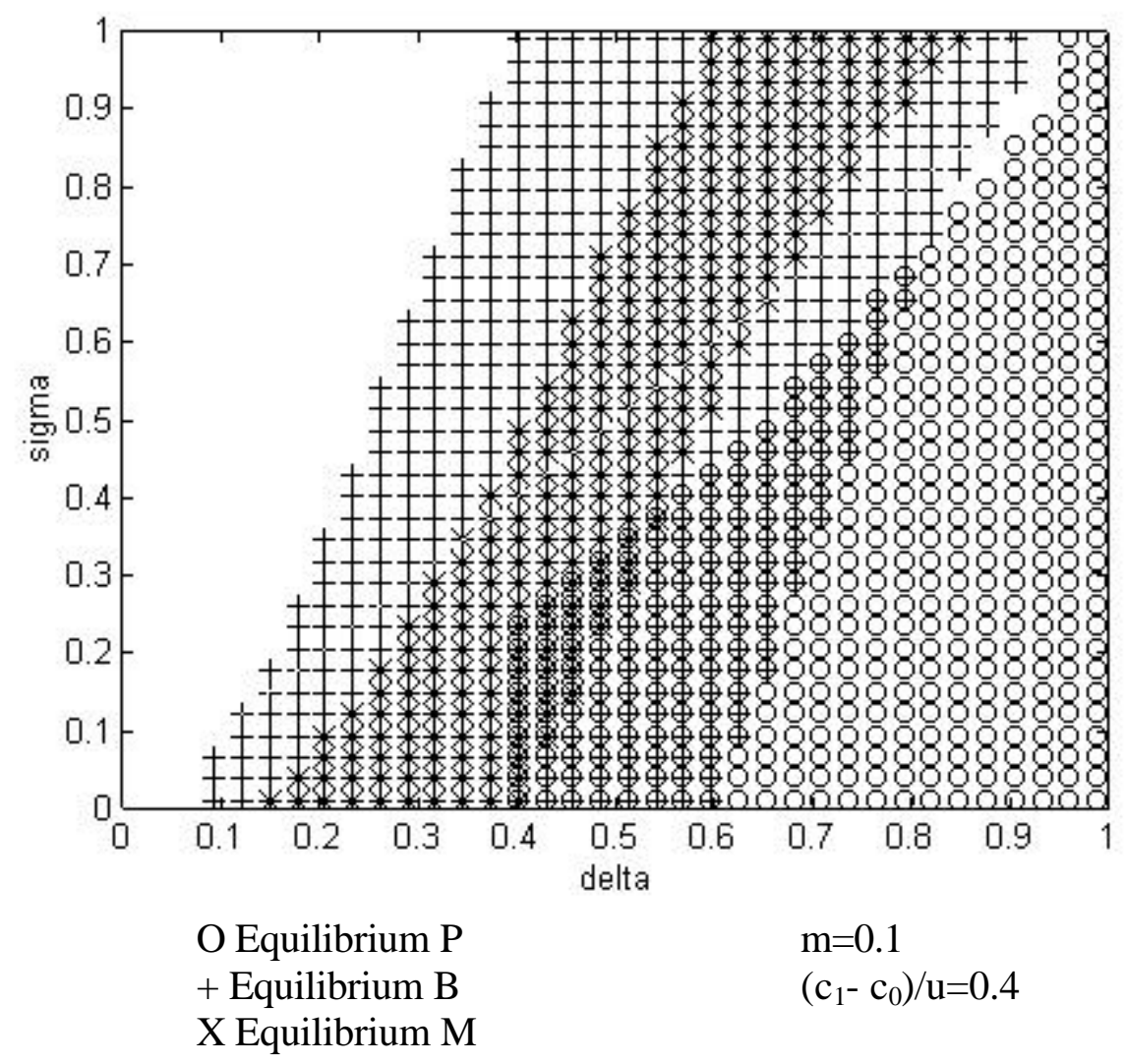


Figure 3

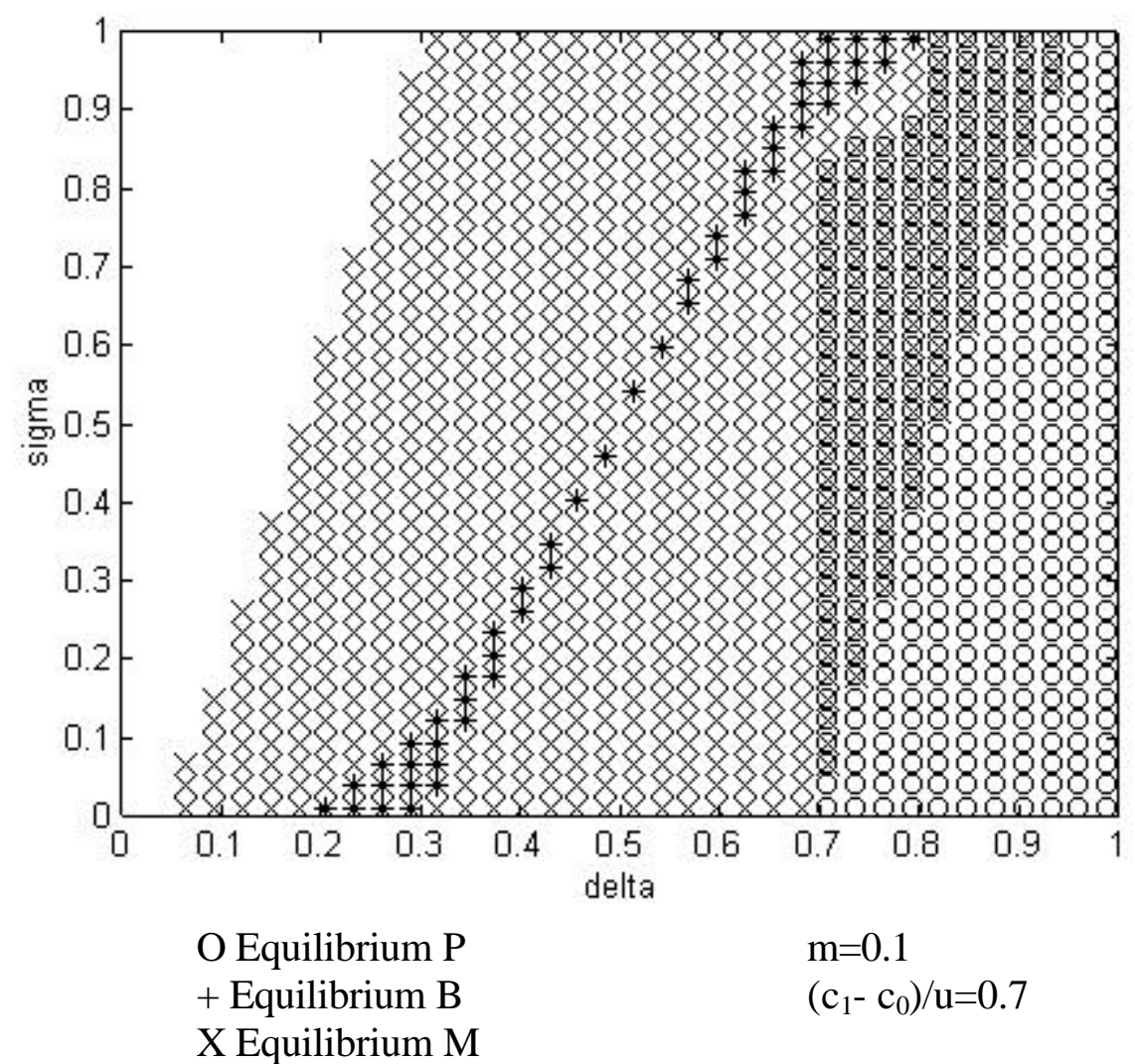


Figure 4

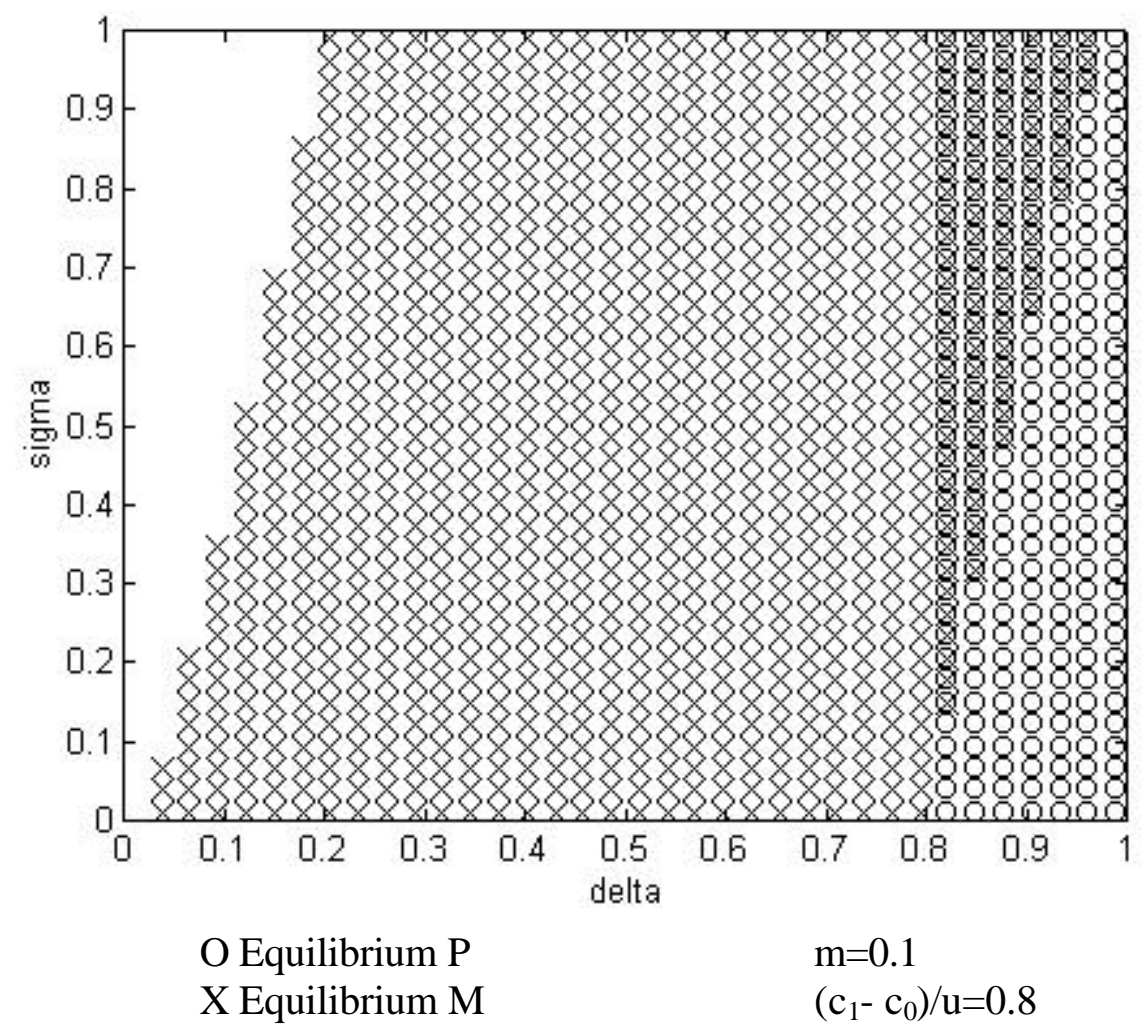


Figure 5

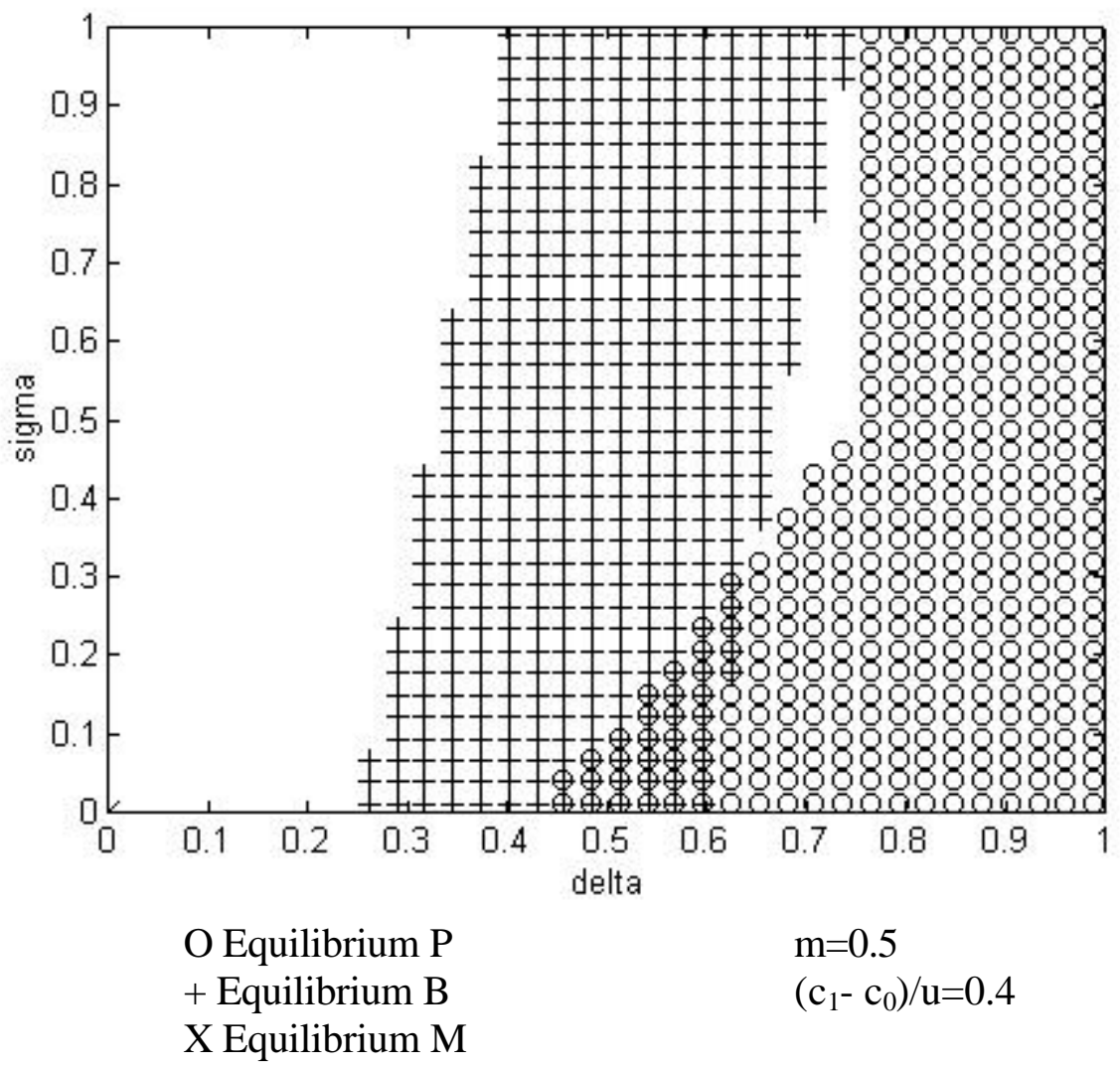


Figure 6

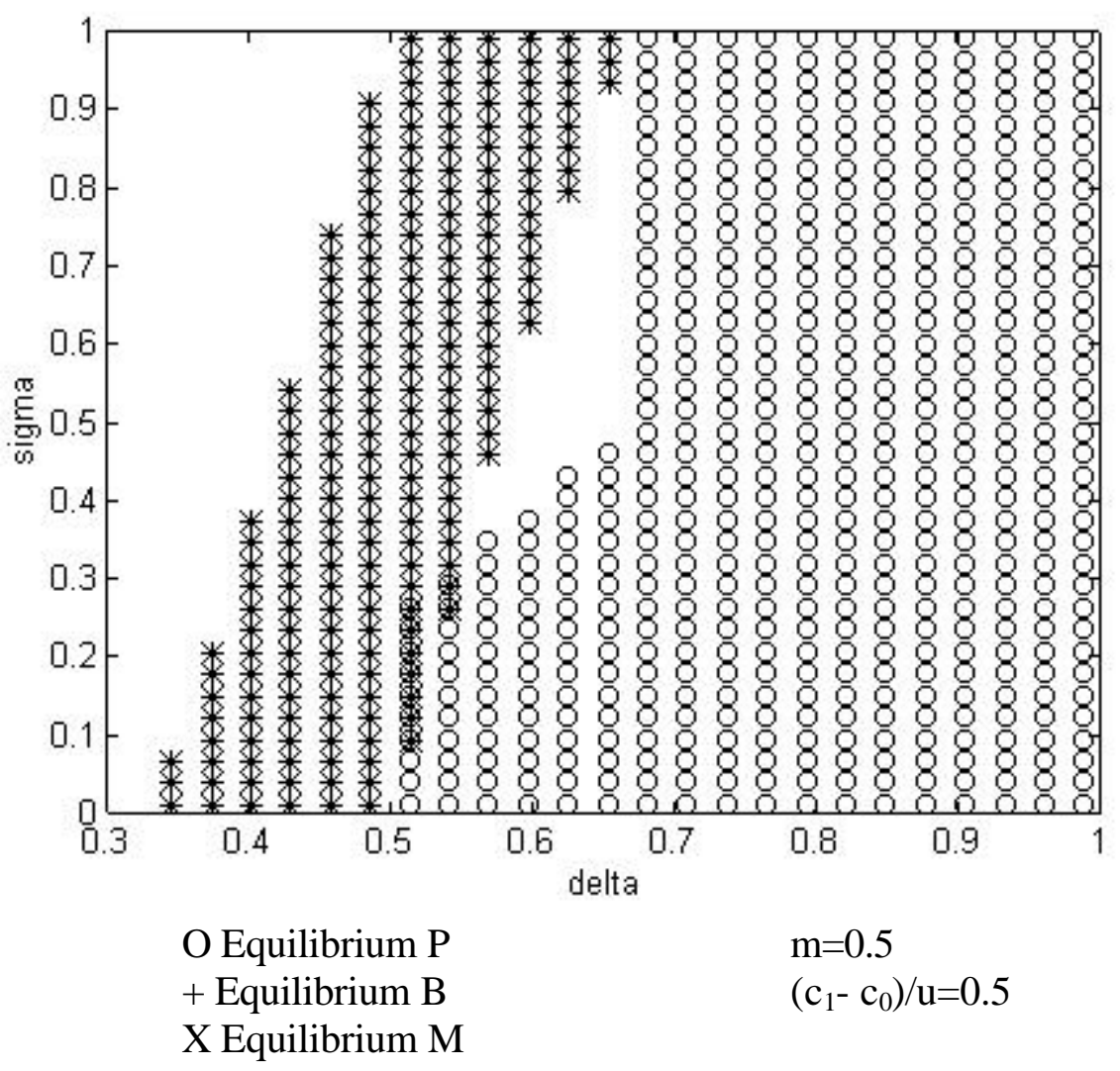


Figure 7

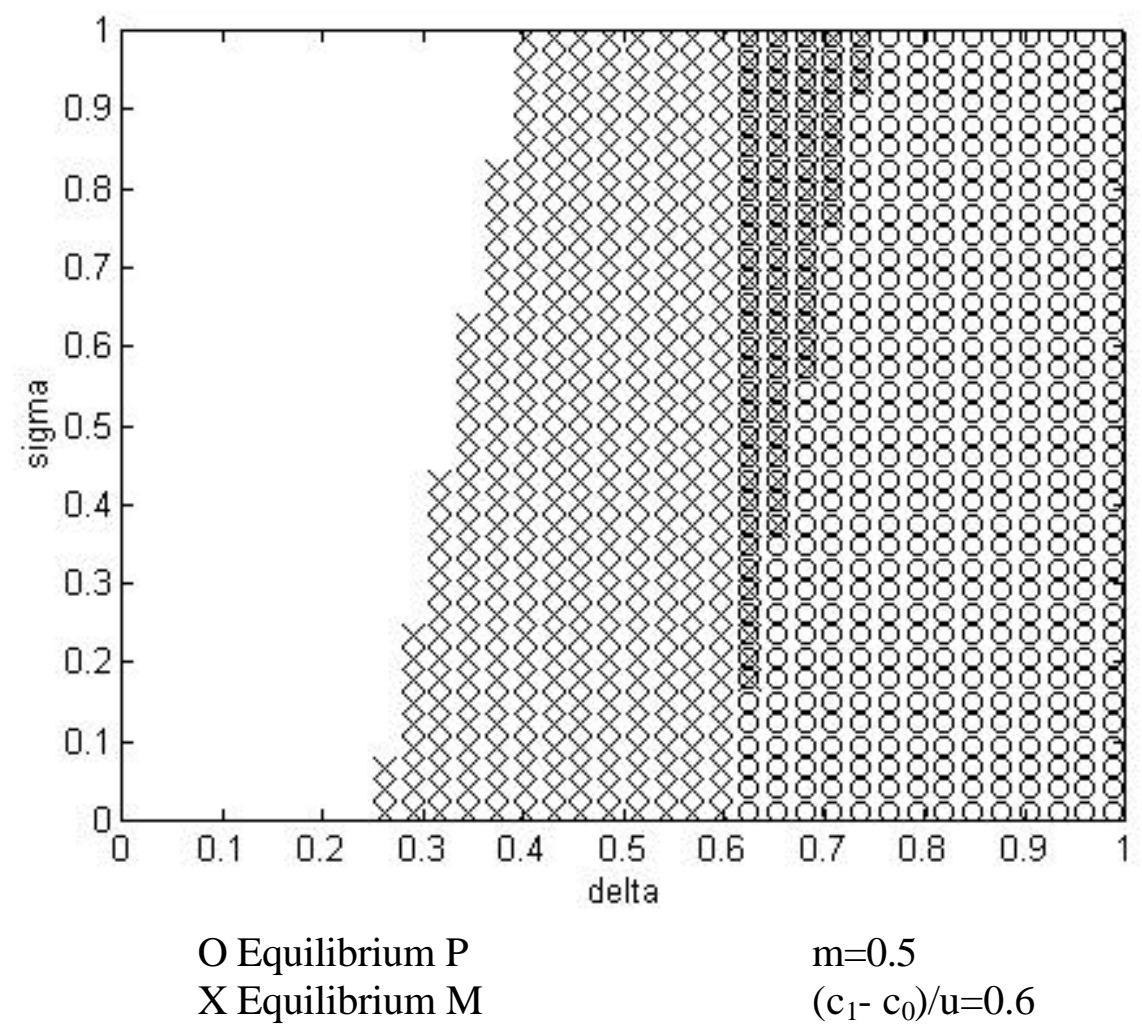


Figure 8

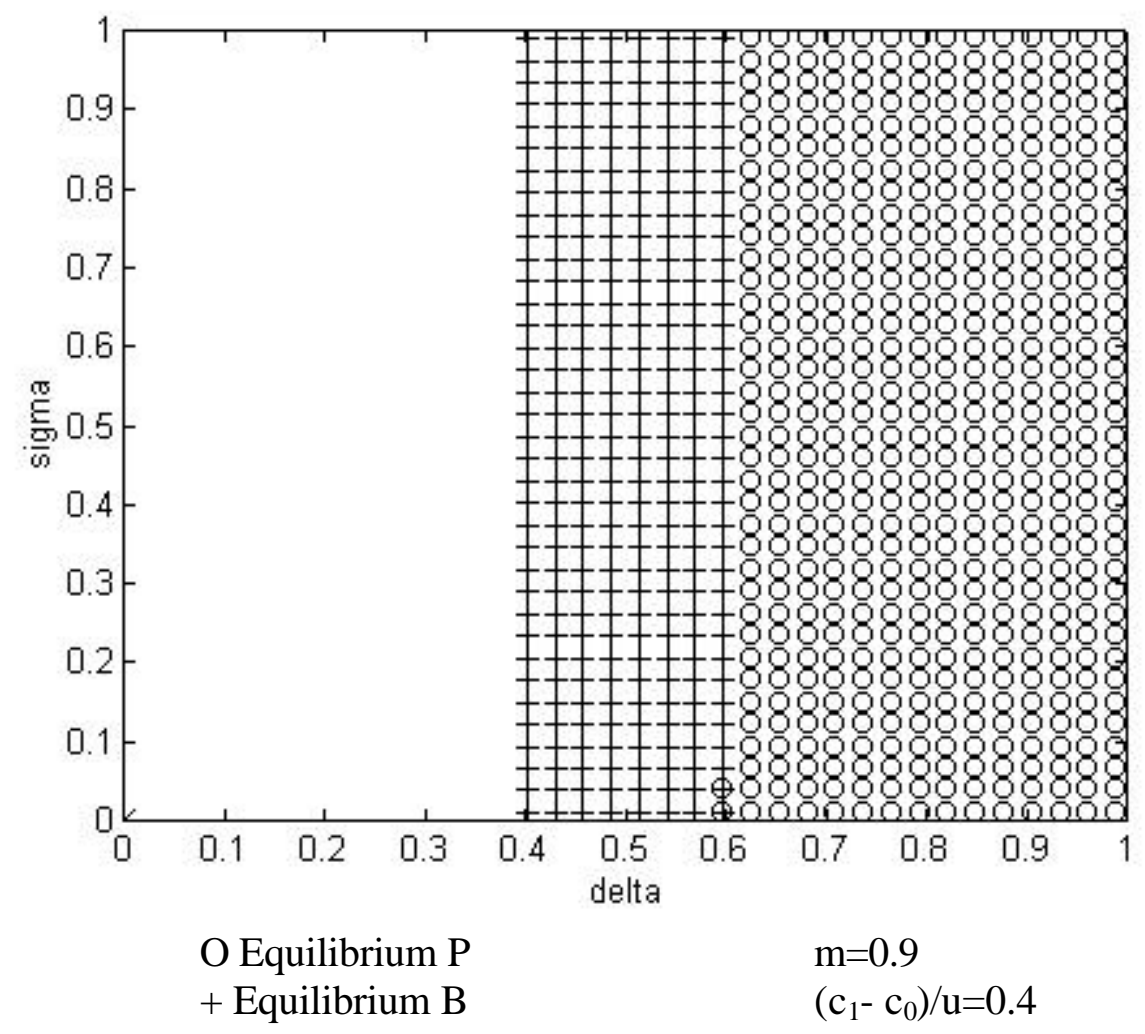


Figure 9

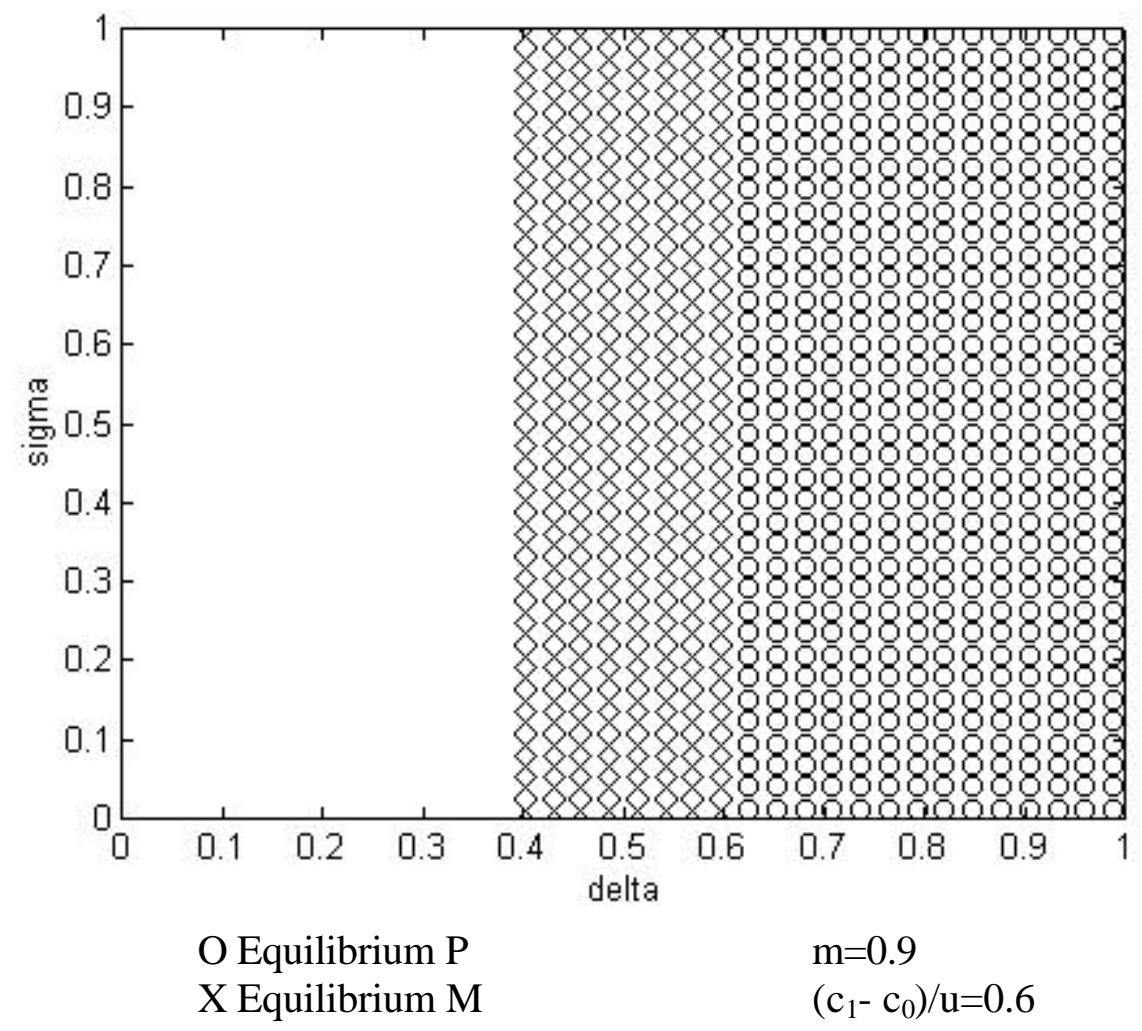


Figure 10

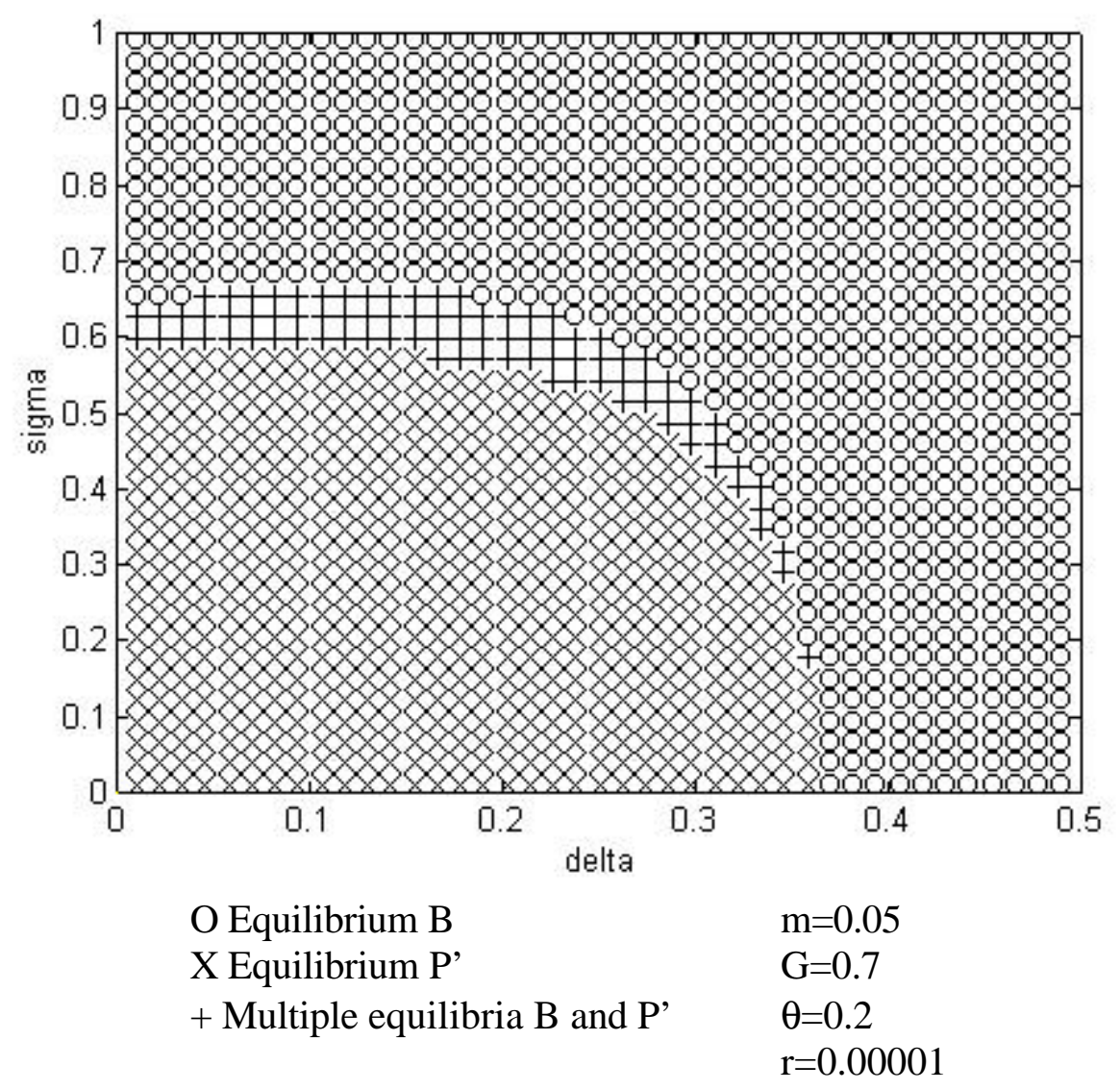


Figure 11

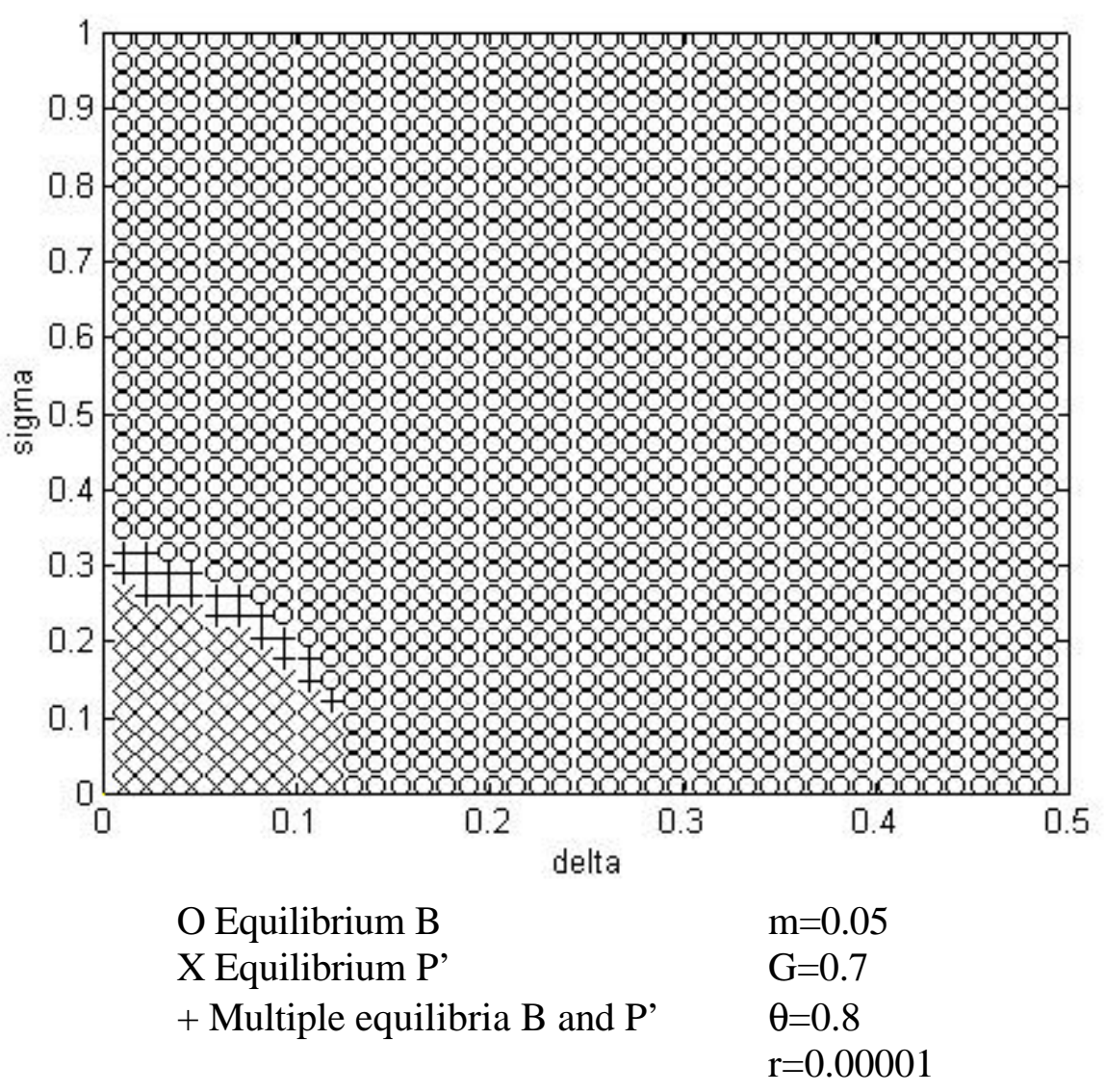


Figure 12

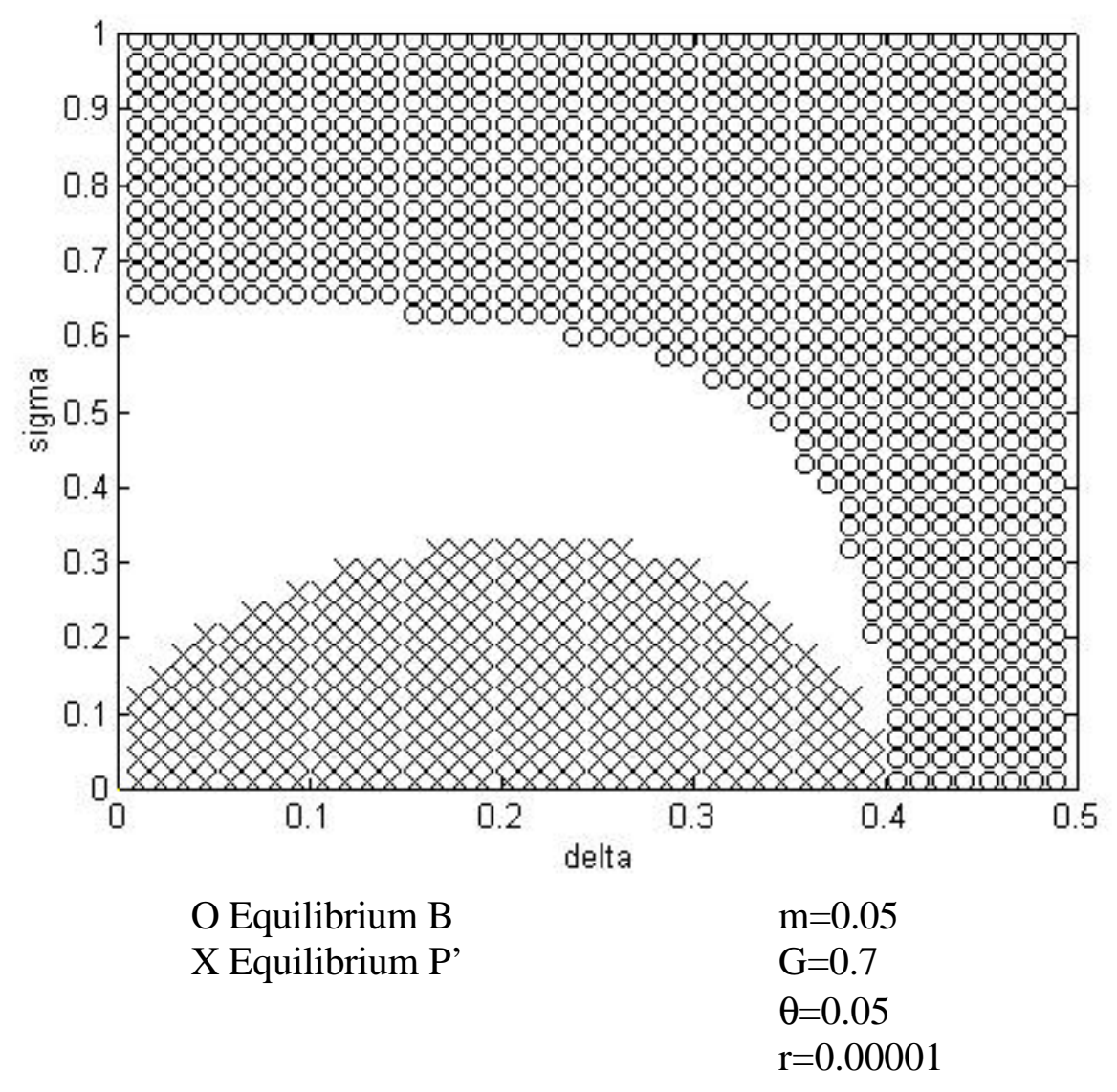


Figure 13

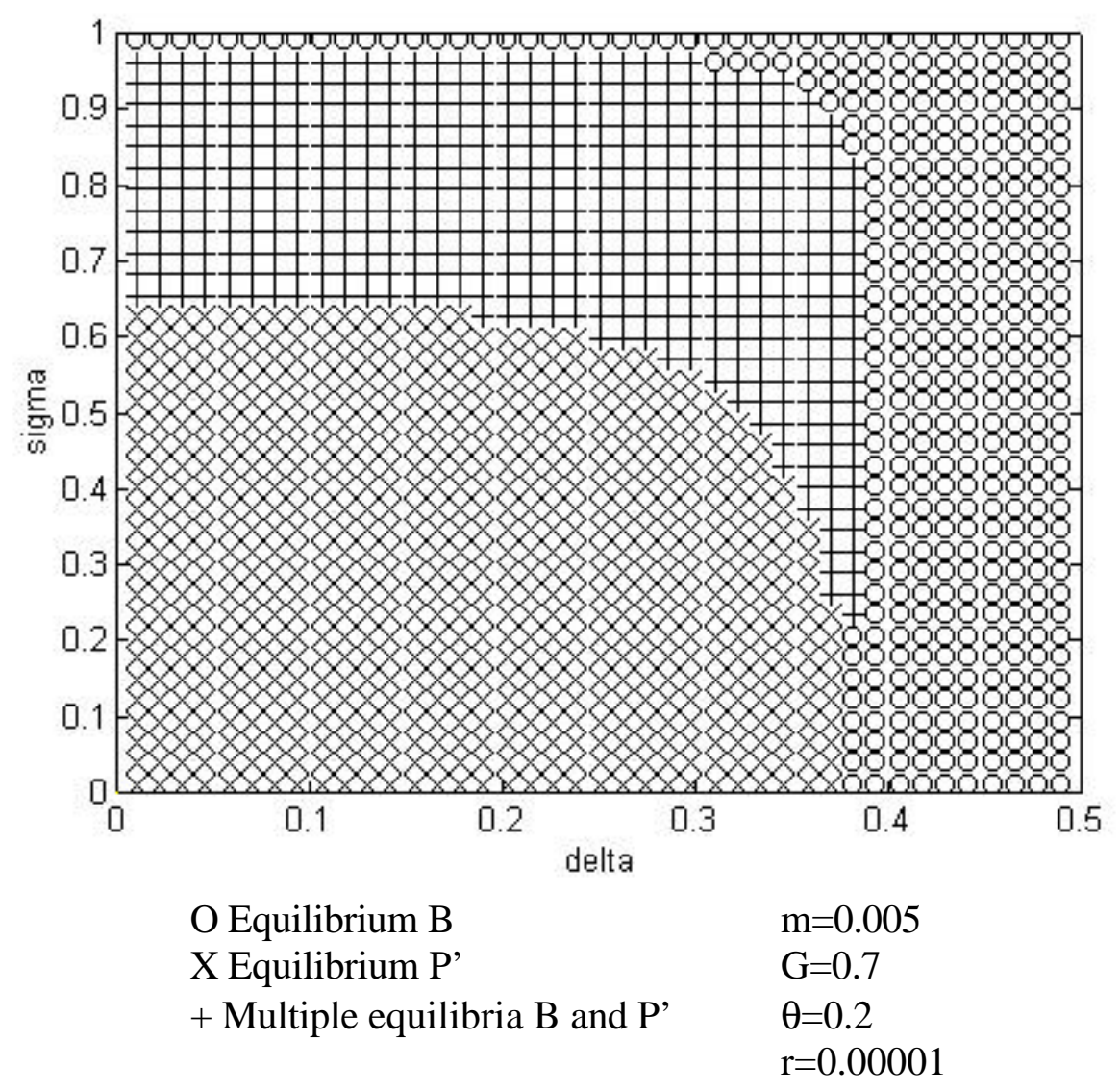


Figure 14

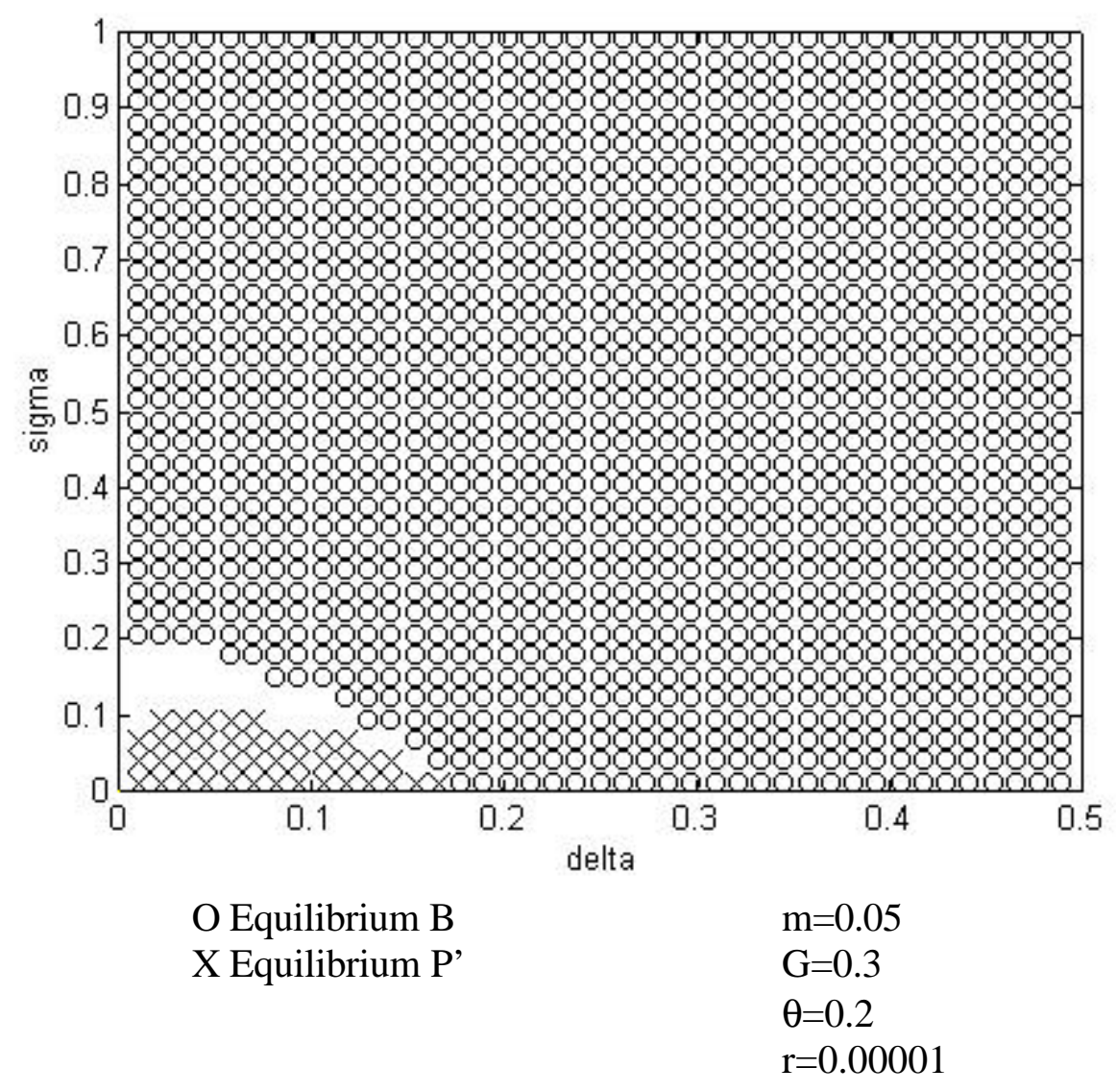

